\title{
Douglas-Square Metrics with Vanishing Mean Stretch Curvature
}

\author{
Akbar Tayebi* and Neda Izadian \\ (Communicated by Yusuf Yaylı)
}

\begin{abstract}
In this paper, we consider the class of square metrics $F=\alpha+2 \beta+\beta^{2} / \alpha$ where $\alpha=\sqrt{a_{i j} y^{i} y^{j}}$ is a Riemannian metric and $\beta=b_{i}(x) y^{i}$ is a one-form on a manifold $M$. Let $(M, F)$ be a Douglas-square manifold. We show that $F$ is a Berwald metric if and only if it a weakly stretch metric. It results that, a Douglas-square metric is R-quadratic if and only if it is a Berwald metric.
\end{abstract}

Keywords: Douglas metric; square metric; stretch metric; Berwald metric;

AMS Subject Classification (2010): Primary: 53B40; Secondary: 53C60.

\section{Introduction}

In 1929, Berwald construct an interesting family of projectively flat Finsler metrics on the unit ball $\mathbb{B}^{n}$ which as follows

$$
F=\frac{\left(\sqrt{\left(1-|x|^{2}\right)|y|^{2}+\langle x, y\rangle^{2}}+\langle x, y\rangle\right)^{2}}{\left(1-|x|^{2}\right)^{2} \sqrt{\left(1-|x|^{2}\right)|y|^{2}+\langle x, y\rangle^{2}}} .
$$

He showed that this class of metrics has constant flag curvature [3]. Berwald's metric can be expressed as

$$
F=\frac{(\alpha+\beta)^{2}}{\alpha}
$$

where

$$
\alpha=\frac{\sqrt{\left(1-|x|^{2}\right)|y|^{2}+\langle x, y\rangle^{2}}}{\left(1-|x|^{2}\right)^{2}}, \quad \beta=\frac{\langle x, y\rangle}{\left(1-|x|^{2}\right)^{2}} .
$$

An Finsler metric in the form (1.2) is called a square metric.

Let $(M, F)$ be a Finsler manifold. In local coordinates, a curve $c(t)$ is a geodesic if and only if its coordinates $\left(c^{i}(t)\right)$ satisfy $\ddot{c}^{i}+2 G^{i}(\dot{c})=0$, where the local functions $G^{i}=G^{i}(x, y)$ are called the spray coefficients. $F$ is called a Berwald metric, if $G^{i}$ are quadratic in $y \in T_{x} M$ for any $x \in M$. In this case, there exists $\Gamma_{j k}^{i}=\Gamma_{j k}^{i}(x)$ such that

$$
G^{i}=\frac{1}{2} \Gamma_{j k}^{i}(x) y^{j} y^{k}
$$

The Douglas metrics are extension of Berwald metrics, which introduced by Douglas as a projective invariant in Finsler geometry. A Finsler metric is called a Douglas metric if

$$
G^{i}=\frac{1}{2} \Gamma_{j k}^{i}(x) y^{j} y^{k}+P(x, y) y^{i}
$$

where $\Gamma_{j k}^{i}=\Gamma_{j k}^{i}(x)$ is a scalar function on $M$ and $P=P(x, y)$ is a homogeneous function of degree one with respect to $y$ on $T M_{0}$. Equivalently, a Finsler metric is a Douglas metric if and only if $G^{i} y^{j}-G^{j} y^{i}$ are 
homogeneous polynomials in $\left(y^{i}\right)$ of degree three [1]. If $P=0$, then $F$ reduces to a Berwald metric. If $\Gamma=0$, then $F$ is a projectively flat Finsler metric.

Other than Douglas metrics, there exist some extensions of Berwald metrics. Let $(M, F)$ be a Finsler manifold. There are two basic tensors on Finsler manifolds: fundamental metric tensor $\mathbf{g}_{y}$ and the Cartan torsion $\mathbf{C}_{y}$, which are second and third order derivatives of $\frac{1}{2} F_{x}^{2}$ at $y \in T_{x} M_{0}$, respectively. The rate of change of the Cartan torsion along geodesics, $\mathbf{L}_{y}$ is said to be Landsberg curvature. Finsler metrics with vanishing Landsberg curvatures are called Landsberg metrics. Every Berwald metric is a Landsberg metric. Taking trace with respect to $\mathbf{g}_{y}$ in first and second variables of $\mathbf{C}_{y}$ and $\mathbf{L}_{y}$ gives rise mean Cartan torsion $\mathbf{I}_{y}$ and mean Landsberg curvature $\mathbf{J}_{y^{\prime}}$, respectively. The mean Landsberg curvature is the rate of change of the mean Cartan torsion along geodesics. As a generalization of Landsberg curvature, Berwald introduced the notion of stretch curvature and denoted it by $\boldsymbol{\Sigma}_{y}$ [4]. He showed that $\boldsymbol{\Sigma}=0$ if and only if the length of a vector remains unchanged under the parallel displacement along an infinitesimal parallelogram. Then, this curvature investigated by Matsumoto in [7]. Taking trace with respect to $\mathbf{g}_{y}$ in first and second variables of $\boldsymbol{\Sigma}_{y}$ gives rise mean stretch curvature $\overline{\boldsymbol{\Sigma}}_{y}$ [9][14][18]. A Finsler metric is said to be weakly stretch metric if $\overline{\boldsymbol{\Sigma}}=0$. By definition, every weakly Landsberg metric is a weakly stretch metric.

In [19], the first author with Tabatabeifar proved that every Douglas-Randers metric with vanishing stretch curvature is a Berwald metric. In this paper, we consider Douglas-square metrics with vanishing mean stretch curvature and obtain the following.

Theorem 1.1. Let $(M, F)$ be a Douglas-square manifold of dimension $n \geq 3$. Then $F$ is a Berwald metric if and only if it is a weakly stretch metric.

A Finsler spaces is said to be R-quadratic if its Riemann curvature $\mathbf{R}_{y}$ is quadratic in $y \in T_{x} M$ [2]. By definition, every Berwald metric is R-quadratic. It is proved that every R-quadratic metric is a stretch metric (see [8][10]). Since every stretch metric is a weakly stretch metric, then by Theorem 1.1 we conclude the following.

Corollary 1.1. Let $F=\alpha+2 \beta+\beta^{2} / \alpha$ be a Douglas metric on a manifold $M$ of dimension $n \geq 3$. Then $F$ is $R$-quadratic if and only if it is a Berwald metric.

There are many connections in Finsler geometry [5][11][12]. Throughout this paper, we use the Cartan connection on Finsler manifolds. The $h$ - and $v$ - covariant derivatives of a Finsler tensor field are denoted by "|" and ", ", respectively.

\section{Proof of Theorem 1.1}

Let $F=\alpha+2 \beta+\beta^{2} / \alpha$ be a square metric on an $n$-dimensional manifold $M$, where $\alpha=\sqrt{a_{i j}(x) y^{i} y^{j}}$ is a Riemannian metric and $\beta(y)=b_{i}(x) y^{i}$ is a 1 -form on $M$. Define $b_{i ; j}$ by $b_{i ; j} \theta^{j}:=d b_{i}-b_{j} \theta_{i}^{j}$, where $\theta^{i}:=d x^{i}$ and $\theta_{i}{ }^{j}:=\gamma_{i k}^{j} d x^{k}$ denote the Levi-Civita connection forms of $\alpha$. Let

$$
\begin{array}{r}
r_{i j}:=\frac{1}{2}\left(b_{i ; j}+b_{j ; i}\right), \quad s_{i j}:=\frac{1}{2}\left(b_{i ; j}-b_{j ; i}\right), s_{j}^{i}:=a^{i h} s_{h j}, \quad s_{j}:=b^{i} s_{i j}, \quad r_{j}:=b^{i} r_{i j}, \\
s_{0}:=s_{i} y^{i}, \quad r_{0}:=r_{i} y^{i}, \quad r_{j 0}:=r_{j i} y^{i}, \quad r_{00}:=r_{i j} y^{i} y^{j}, \quad s_{0}^{i}:=s_{j}^{i} y^{j},
\end{array}
$$

where $b^{i}:=a^{i j} b_{j}$ and $\left(a^{i j}\right)=\left(a_{i j}\right)^{-1}$.

To finding the relation between the Levi-Civita and Cartan connections of $\alpha$ and $F$, we put the difference tensor $D_{j k}^{i}:=\Gamma_{j k}^{i}-\gamma_{j k}^{i}$, where $\Gamma_{j k}^{i}$ and $\gamma_{j k}^{i}$ denoted the Cheristoffel symbols of the Cartan connection of $F$ and the Levi-Civita connection of $\alpha$, respectively. The tensor $D_{i k}^{j}$ is called the difference tensor which is computed by Matsumoto in [7]. Now, let $\nabla_{k}$ be the covariant differentiation by $x^{k}$ with respect to associated Riemannian connection. Let us put

$$
b_{i ; j}:=\nabla_{j} b_{i}:=\frac{\partial b_{i}}{\partial x^{j}}-b_{r} \gamma_{i j}^{r}, \quad r_{i j k}:=\nabla_{k} r_{i j}, \quad s_{i j k}:=\nabla_{k} s_{i j} .
$$

We recall that the index 0 mean contraction by $y^{i}$. For example $r_{0 j k}=r_{i j k} y^{i}$.

For a square metric $F=\alpha+2 \beta+\beta^{2} / \alpha$, the following holds

$$
G^{i}=G_{\alpha}^{i}+\frac{2 \alpha}{1-s} s_{0}^{i}+\frac{\theta}{1-s}\left\{(1-s) r_{00}-4 \alpha s_{0}\right\}\left\{\frac{y^{i}}{\alpha}+\chi b^{i}\right\}
$$


where $G^{i}$ and $G_{\alpha}^{i}$ are the spray coefficients of $F$ and $\alpha$, respectively, and

$$
\theta:=\frac{1-2 s}{-3 s^{2}+2 b^{2}+1}, \quad \chi:=\frac{1}{1-2 s}, \quad s:=\frac{\beta}{\alpha}
$$

and $b^{2}:=\|\beta\|_{\alpha}=\sqrt{a_{i j} b^{i} b^{j}}$ (see [13], [15], [16] and [20]).

In [6], Li-Shen-Shen characterized $(\alpha, \beta)$-metrics with vanishing Douglas curvature. In particular, we will use the following.

Lemma 2.1. Let $F=\alpha+2 \beta+\beta^{2} / \alpha$ be a square metric on an open subset $U \subset \mathbb{R}^{n}(n \geq 3)$. Then $F$ is a Douglas metric if and only if

$$
b_{i ; j}=2 \tau\left\{\left(1+2 b^{2}\right) a_{i j}-3 b_{i} b_{j}\right\}
$$

where $\tau=\tau(x)$ is a scalar function on $U$.

Put

$$
D^{i}:=2\left(G^{i}-G_{\alpha}^{i}\right),
$$

By assumption, the square metric $F=\alpha+2 \beta+\beta^{2} / \alpha$ has vanishing Douglas curvature. Then, by Lemma 2.1, $\beta$ is a close 1 -form, i.e., $s_{i j}=0$. In this case, by (2.2) we get

$$
D^{i}=2 \theta\left(\frac{y^{i}}{\alpha}+\chi b^{i}\right) r_{00} .
$$

Taking a vertical derivative of (2.4) yields

$$
D_{j}^{i}=B y^{i} b_{j}+C y^{i} y_{j}-D \delta_{j}^{i}-E y^{i} r_{0 j}+G b^{i} b_{j}-H b^{i} y_{j}+K b^{i} r_{0 j},
$$

where

$$
\begin{aligned}
B & :=\frac{-4\left(2 \alpha^{2} b^{2}+3 \beta^{2}-3 \alpha \beta+\alpha^{2}\right) r_{00}}{\left(2 \alpha^{2} b^{2}-3 \beta^{2}+\alpha^{2}\right)^{2}} \\
C & :=\frac{2\left[2 \beta\left(2 \alpha^{2} b^{2}+3 \beta^{2}-3 \alpha \beta+\alpha^{2}\right)+(2 \beta-\alpha)\left(2 \alpha^{2} b^{2}-3 \beta^{2}+\alpha^{2}\right)\right] r_{00}}{\alpha^{2}\left(2 \alpha^{2} b^{2}-3 \beta^{2}+\alpha^{2}\right)^{2}} \\
D & :=\frac{2(2 \beta-\alpha) r_{00}}{\left(2 \alpha^{2} b^{2}-3 \beta^{2}+\alpha^{2}\right)} \\
E & :=\frac{4(2 \beta-\alpha)}{\left(2 \alpha^{2} b^{2}-3 \beta^{2}+\alpha^{2}\right)} \\
G & :=\frac{12 \alpha^{2} \beta r_{00}}{\left(2 \alpha^{2} b^{2}-3 \beta^{2}+\alpha^{2}\right)^{2}} \\
H & :=\frac{12 \beta^{2} r_{00}}{\left(2 \alpha^{2} b^{2}-3 \beta^{2}+\alpha^{2}\right)^{2}} \\
K & :=\frac{4 \alpha^{2}}{\left(2 \alpha^{2} b^{2}-3 \beta^{2}+\alpha^{2}\right)} .
\end{aligned}
$$

By contracting (2.5) with $b_{i}$, we have

$$
b_{i} D_{j}^{i}=\left(B \beta-D+G b^{2}\right) b_{j}+\left(C \beta-H b^{2}\right) y_{j}+\left(K b^{2}-E \beta\right) r_{0 j} .
$$

Putting $b_{i \mid j}=b_{i ; j}-b_{r} D_{i j}^{r}$ in (2.6) yields

$$
b_{i \mid 0}=-\left(B \beta-D+G b^{2}\right) b_{i}-\left(C \beta-H b^{2}\right) y_{i}+\left(1+E \beta-K b^{2}\right) r_{0 i} .
$$

Multiplying (2.7) with $y^{i}$ implies that

$$
b_{0 \mid 0}=-\left(B \beta-D+G b^{2}\right) \beta-\left(C \beta-H b^{2}\right) \alpha^{2}+\left(1+E \beta-K b^{2}\right) r_{00} .
$$


On the other hand, the mean Cartan torsion of a square metric $F=\alpha+2 \beta+\beta^{2} / \alpha$ is given by

$$
I_{i}:=-\frac{1}{2 \alpha} A\left(b_{i}-\alpha^{-2} \beta y_{i}\right),
$$

where

$$
A:=\frac{2(n-2) s}{1-s^{2}}-\frac{2(n+1)}{1+s}+\frac{6 s}{1-3 s^{2}+2 b^{2}} .
$$

By (2.9) we have

$$
J_{i}=\frac{1}{2 \alpha^{4}}\left(A \alpha_{\mid 0}-A_{\mid 0} \alpha\right)\left(\alpha^{2} b_{i}-\beta y_{i}\right)-\frac{A}{2 \alpha} b_{i \mid 0}+\frac{A}{2 \alpha^{4}}\left(\alpha b_{0 \mid 0}-2 \beta \alpha_{\mid 0}\right) y_{i}+\frac{A \beta}{2 \alpha^{3}} y_{i \mid 0} .
$$

By putting (2.7) and (2.8) in (2.10), one can get the following

$$
J_{i}=X b_{i}+Y y_{i}-W r_{0 i}
$$

where $X, Y$ and $W$ are listed in Appendix. By taking a horizontal derivation of (2.11) along a geodesic and contracting the result with $b^{i}$, we get

$$
b^{i} J_{i \mid 0}=\gamma\left(b_{1} r_{00 \mid 0}+b_{2} r_{0} r_{00}+b_{3} r_{00}^{2}+b_{4} b^{i} r_{0 i \mid 0}+b_{5} r_{0}+b_{6} r_{0}^{2}+b_{7} r_{0 \mid 0}+b_{8} r_{00}\right),
$$

where $b_{i}(1 \leq i \leq 8)$ are listed in Appendix, and

$$
\gamma:=\frac{1}{\alpha^{2}\left(\alpha^{2}-\beta^{2}\right)^{2}\left(2 \alpha^{2} b^{2}+\alpha^{2}-3 \beta^{2}\right)^{5}} .
$$

By some computations, we get

$$
\begin{gathered}
r_{0 \mid 0}=b^{i} r_{i 0 \mid 0}, \\
r_{i 0 \mid 0}=r_{i 00}-r_{i m} D_{00}^{m}-r_{m 0} D_{i 0}^{m}, \\
r_{00 \mid 0}=\operatorname{A}\left(4 \alpha^{5} b^{2} r_{00}^{2}+2 \alpha^{5} r_{00}^{2}+4 \alpha^{4} b^{4} r_{000}-8 \alpha^{4} b^{2} \beta r_{00}-8 \alpha^{4} b^{2} r_{0} r_{00}-6 r_{00}^{2} \alpha^{3}\right. \\
-18 \alpha^{3} \beta^{2} r_{00}^{2}+24 \alpha^{2} \beta^{3} r_{00}^{2}+4 \alpha^{4} b^{2} r_{000}-4 \alpha^{4} \beta r_{00}-4 \alpha^{4} r_{0} r_{00}-12 \alpha^{3} b^{2} r_{00}^{2} \\
-12 \alpha^{3} \beta^{2} r_{00}-6 \alpha^{2} \beta^{2} r_{000}-12 \alpha^{2} b^{2} \beta^{2} r_{000}+20 \alpha^{2} b^{2} \beta r_{00}^{2}+12 \alpha^{2} \beta^{3} r_{00} \\
+\alpha^{4} r_{000}+12 \alpha^{2} \beta^{2} r_{0} r_{00}+9 \beta^{4} r_{000}+10 \alpha^{2} \beta r_{00}^{2} \\
\left.+18 \alpha \beta^{2} r_{00}^{2}-30 \beta^{3} r_{00}^{2}\right),
\end{gathered}
$$

where

$$
\mathfrak{A}:=\frac{1}{\left(2 b^{2} \alpha^{2}+\alpha^{2}-3 \beta^{2}\right)^{2}}
$$

Thus, we have

$$
\begin{aligned}
& b^{i} r_{i 0 \mid 0}=b^{i} r_{i 00}+\mathfrak{A}\left(8 \alpha^{4} b^{4} r_{00}^{2}-12 \alpha^{4} b^{2} \beta r_{0} r_{00}+4 \alpha^{4} b^{4} b^{i} r_{i 00}-4 \alpha^{4} b^{2} r_{0}^{2}-4 \alpha^{4} b^{2} r_{0} r_{00}\right. \\
& +4 \alpha^{4} b^{2} r_{00}^{2}-8 \alpha^{3} b^{2} \beta r_{00}^{2}+4 \alpha^{2} b^{2} \beta^{2} r_{00}^{2}+12 \beta^{3} r_{00} \alpha^{2} r_{0}-12 \alpha^{2} b^{2} \beta^{2} b^{i} r_{i 00} \\
& -4 \alpha^{2} \beta^{2} r_{00}^{2}-6 \alpha \beta^{3} r_{00}^{2}+4 \alpha^{4} b^{2} b^{i} r_{i 00}-2 \alpha^{4} r_{0}^{2}-2 \alpha^{4} r_{0} r_{00}-4 \alpha^{3} b^{2} r_{0} r_{00} \\
& -2 \alpha^{3} r_{0} r_{00}-8 \alpha^{2} b^{2} \beta^{2} r_{00}+4 \alpha^{2} b^{2} \beta r_{0} r_{00}+6 \alpha^{2} \beta^{2} r_{0}^{2}-4 \alpha^{2} \beta^{2} r_{00} \\
& +6 \alpha \beta^{2} r_{0} r_{00}+9 \beta^{4} b^{i} r_{i 00}-6 \alpha^{2} \beta^{2} b^{i} r_{i 00}+6 \alpha^{2} \beta^{2} r_{0} r_{00}+2 \alpha^{2} \beta r_{0} r_{00} \\
& \left.+\alpha^{4} b^{i} r_{i 00}-12 \alpha \beta^{3} r_{00}+12 \beta^{4} r_{00}^{2}+2 \alpha^{3} \beta r_{00}^{2}+12 \beta^{4} r_{00}-6 \beta^{3} r_{0} r_{00}\right) \text {. }
\end{aligned}
$$

By definition of the Cartan connection, we have $g_{i j \mid s}=0$ and then $g_{\mid s}^{i j}=0$. Therefore, we have

$$
\bar{\Sigma}_{k l}=g^{i j} \Sigma_{i j k l}=g^{i j}\left(L_{i j k \mid l}-L_{i j l \mid k}\right)=J_{k \mid l}-J_{l \mid k} .
$$


By assumption, $F$ is a weakly stretch metric $\bar{\Sigma}=0$. Thus $J_{i \mid k}=J_{k \mid i}$. Contracting it with $y^{k}$ yields $J_{i \mid k} y^{k}=0$ or $J_{i \mid 0}=0$. This equation is equivalent to that for any linearly parallel vector field $u$ along a geodesic $c$, the following holds:

$$
\frac{d}{d t}\left[\mathbf{J}_{\dot{c}}(u)\right]=0
$$

The geometric meaning of this is that the rate of change of the mean Landsberg curvature is constant along any Finslerian geodesic.

Since $J_{i \mid 0}=0$, then $b^{i} J_{i \mid 0}=0$. Then by putting (2.13), (2.14), (2.15) and (2.16) in (2.12), we get

$$
\gamma\left(A_{16} \alpha^{16}+\cdots+A_{1} \alpha+A_{0}\right)=0 .
$$

where $A_{i}(0 \leq i \leq 16)$ are given in the Appendix. By (2.17) we get

$$
\begin{aligned}
& A_{16} \alpha^{16}+A_{14} \alpha^{14}+\cdots+A_{2} \alpha^{2}+A_{0}=0 \\
& A_{15} \alpha^{14}+A_{13} \alpha^{12}+\cdots+A_{3} \alpha^{2}+A_{1}=0 .
\end{aligned}
$$

By (2.18) and (2.19) we get

$$
\begin{aligned}
& 3(3 n+10) \beta r_{000}+(97 n+350) r_{00}^{2}=f \alpha^{2}, \\
& 3(4 n-10) \beta r_{000}-(353 n+759) r_{00}^{2}=g \alpha^{2},
\end{aligned}
$$

where $f=f(x, y)$ and $g=g(x, y)$ are homogeneous functions of degree 2 with respect to $y$ on $T M$. (2.21) implies that

$$
r_{000}=h \alpha^{2}+k r_{00}^{2}
$$

where $h=h(x, y)$ and $k=k(x, y)$ are homogeneous functions of degree 1 with respect to $y$ on $T M$. Putting (2.22) in (2.20) yields

$$
r_{00}=t \alpha^{2}
$$

where $t=t(x, y)$ is a homogeneous function of degree 0 with respect to $y$ on $T M$.

By Lemma 2.1, the following holds

$$
b_{i ; j}=2 \tau\left\{\left(1+2 b^{2}\right) a_{i j}-3 b_{i} b_{j}\right\}
$$

where $\tau=\tau(x)$ is a scalar function. We claim that $\tau=0$. On contrary, suppose that $\tau \neq 0$. Thus by (2.24), we get

$$
r_{i j}=2 \tau\left\{\left(1+2 b^{2}\right) a_{i j}-3 b_{i} b_{j}\right\} \text {. }
$$

which yields

By (2.23) and (2.26), we get

$$
r_{00}=2 \tau\left\{\left(1+2 b^{2}\right) \alpha^{2}-3 \beta^{2}\right\} .
$$

$$
\alpha^{2}=\left[\frac{6 \tau}{2 \tau\left(1+2 b^{2}\right)-t}\right] \beta^{2} .
$$

This contradicts with the positive-definiteness of $\alpha$. Then $\tau=0$. By considering (2.24), it follows that $\beta$ is parallel with respect to $\alpha$. Therefore, $F$ reduces to a Berwald metric.

\section{Appendix}

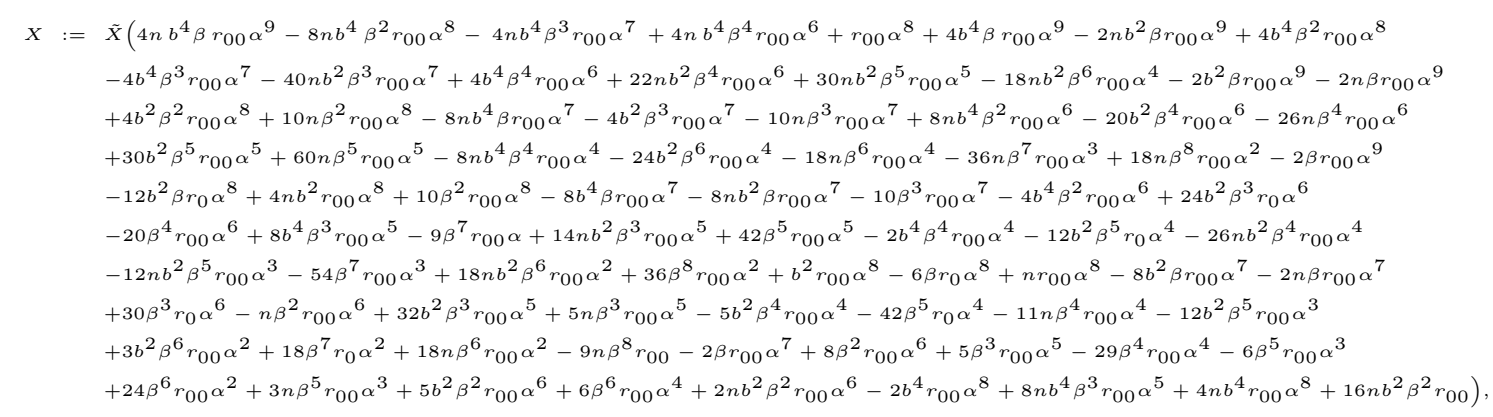


$Y:=\tilde{Y}\left(8 b^{4} \beta n r_{00} \alpha^{10}-8 n b^{4} \beta^{2} r_{00} \alpha^{9}-16 b^{4} \beta^{5} \alpha^{6}+8 n b^{4} \beta^{5} r_{00} \alpha^{6}+8 b^{4} \beta r_{00} \alpha^{10}+8 n b^{2} \beta r_{00} \alpha^{8}-16 n b^{4} \beta^{2} \alpha^{5}+16 b^{4} \beta^{2} r_{00} \alpha^{9}\right.$ $-8 n b^{4} \beta r_{00} \alpha^{9}-20 n b^{2} \beta^{2} r_{00} \alpha^{9}+24 n b^{4} \beta^{2} r_{00} \alpha^{8}-\alpha^{9} r_{00}-24 n \alpha^{8} b^{2} r_{00} \beta^{3}+16 n b^{4} \beta^{4} \alpha^{7}-8 n b^{4} \beta^{3} r_{00} \alpha^{7}+72 n b^{2} \beta^{4} r_{00} \alpha^{7}$ $+8 b^{4} \beta^{5} r_{00} \alpha^{6}-16 n b^{4} \beta^{4} r_{00} \alpha^{6}-4 n b^{2} \beta^{5} r_{00} \alpha^{6}-36 n b^{2} \beta^{6} r_{00} \alpha^{5}+12 n b^{2} \beta^{7} r_{00} \alpha^{4}+8 b^{2} \beta r_{00} \alpha^{10}+2 n \beta r_{00} \alpha^{10}-16 b^{4} \beta^{2} \alpha^{9}$ $+8 n b^{4} \beta^{5} r_{00} \alpha^{5}-4 n b^{4} r_{00} \alpha^{9}-16 n b^{2} \beta^{2} \alpha^{9}-8 b^{2} r_{00} \beta^{2} \alpha^{9}-8 n b^{2} \beta r_{00} \alpha^{9}-8 n \beta^{2} r_{00} \alpha^{9}-16 b^{4} \beta^{3} \alpha^{8}+8 n b^{2} \beta^{3} \alpha^{8}$ $+24 n b^{2} \beta^{2} r_{00} \alpha^{8}-6 n \beta^{3} r_{00} \alpha^{9}+16 b^{4} \beta^{4} \alpha^{7}+16 n b^{4} \beta^{3} r_{00} \alpha^{7}+48 n b^{4} \beta^{2} r_{00} \alpha^{7}+112 n b^{2} \beta^{4} \alpha^{7}+24 b^{2} \beta^{4} r_{00} \alpha^{7}+28 n b^{2} \beta^{3} r_{00} \alpha^{7}$ $+54 n \beta^{4} r_{00} \alpha^{7}-16 b^{4} \beta^{4} r_{00} \alpha^{6}-28 n b^{4} \beta^{3} r_{00} \alpha^{6}-88 n b^{2} \beta^{5} \alpha^{6}+56 b^{2} \beta^{5} r_{00} \alpha^{6}-124 n b^{2} \beta^{4} r_{00} \alpha^{6}-22 n \beta^{5} r_{00} \alpha^{6}+8 b^{4} \beta^{5} r_{00} \alpha^{5}$ $-28 n b^{4} \beta^{4} r_{00} \alpha^{5}-48 b^{2} \beta^{6} r_{00} \alpha^{5}+44 n b^{2} \beta^{5} r_{00} \alpha^{5}-18 n \beta^{6} r_{00} \alpha^{5}+b^{4} \beta^{5} r_{00} \alpha^{4}+48 n b^{2} \beta^{7} \alpha^{4}+72 n b^{2} \beta^{6} r_{00} \alpha^{4}+78 n \beta^{7} r_{00} \alpha^{4}$ $+2 \beta r_{00} \alpha^{10}-36 n b^{2} \beta^{7} r_{00} \alpha^{3}+54 n \beta^{8} r_{00} \alpha^{3}-36 n \beta^{9} r_{00} \alpha^{2}-4 b^{4} r_{00} \alpha^{9}-16 b^{2} \beta^{2} \alpha^{9}-8 b^{2} \beta r_{00} \alpha^{9}-4 n b^{2} r_{00} \alpha^{9}-4 n \beta^{2} \alpha^{9}$ $-8 \beta^{2} r_{00} \alpha^{9}-2 n \beta r_{00} \alpha^{9}-24 b^{4} \beta r_{00} \alpha^{8}-16 b^{2} \beta^{3} \alpha^{8}-24 b^{2} \beta^{2} r_{0} \alpha^{8}-6 \beta^{3} r_{00} \alpha^{8}+6 n \beta^{2} r_{00} \alpha^{8}+40 b^{2} \beta^{4} \alpha^{7}+40 b^{2} \beta^{3} r_{00} \alpha^{7}$ $+52 n \beta^{4} \alpha^{7}+48 \beta^{4} r_{00} \alpha^{7}+16 n \beta^{3} r_{00} \alpha^{7}+32 b^{4} \beta^{3} r_{00} \alpha^{6}+8 b^{2} \beta^{5} \alpha^{6}+48 b^{2} \beta^{4} r_{0} \alpha^{6}-40 b^{2} \beta^{4} r_{00} \alpha^{6}-28 n b^{2} \beta^{3} r_{00} \alpha^{6}-4 n \beta^{5} \alpha^{6}$ $-58 n \beta^{4} r_{00} \alpha^{6}-28 b^{4} r_{00} \beta^{4} \alpha^{5}-72 b^{2} \beta^{6} \alpha^{5}-40 b^{4} \beta^{5} r_{00} \alpha^{5}-172 n b^{2} \beta^{4} r_{00} \alpha^{5}-144 n \beta^{6} \alpha^{5}-96 \beta^{6} r_{00} \alpha^{5}+24 b^{4} \beta^{5} r_{00} \alpha^{4}$ $-24 b^{2} \beta^{6} r_{0} \alpha^{4}+84 b^{2} \beta^{6} r_{00} \alpha^{4}+96 n b^{2} \beta^{5} r_{00} \alpha^{4}+54 \beta^{7} r_{00} \alpha^{4}+144 n \beta^{6} r_{00} \alpha^{4}-48 b^{2} \beta^{7} r_{00} \alpha^{3}+84 n b^{2} \beta^{6} r_{00} \alpha^{3}+72 n \beta^{8} \alpha^{3}$ $-36 n \beta^{9} \alpha^{2}-72 \beta^{9} r_{00} \alpha^{2}-72 n \beta^{8} r_{00} \alpha^{2}+36 \beta^{9} r_{00} \alpha-4 \alpha^{9} b^{2} r_{00}-4 \beta^{2} \alpha^{9}-2 \beta r_{00} \alpha^{9}-n r_{00} \alpha^{9}-12 b 2 \beta r_{00} \alpha^{8}-4 \beta^{3} \alpha^{8}$ $-36 n b^{2} \beta^{7} r_{00} \alpha^{2}+6 \beta^{2} r_{00} \alpha^{8}+36 b^{2} \beta^{2} r_{00} \alpha^{7}+52 \beta^{4} \alpha^{7}+16 \beta^{3} r_{00} \alpha^{7}+18 n \beta^{2} r_{00} \alpha^{7}+44 b^{2} \beta^{3} r_{00} \alpha^{6}+8 \beta^{5} \alpha^{6}+60 \beta^{4} r_{0} \alpha^{6}$ $-7 n \beta^{3} r_{00} \alpha^{6}-76 b^{2} r_{00} \beta^{4} \alpha^{5}-108 \beta^{6} \alpha^{5}-22 \beta^{5} r_{00} \alpha^{5}-88 n \beta^{4} r_{00} \alpha^{5}+12 b^{2} \beta^{5} r_{00} \alpha^{4}+36 \beta^{7} \alpha^{4}-84 \beta^{6} r_{0} \alpha^{4}+114 \beta^{6} r_{00} \alpha^{4}$ $+45 n \beta^{5} r_{00} \alpha^{4}+108 b^{2} \beta^{6} r_{00} \alpha^{3}-24 \beta^{7} r_{00} \alpha^{3}+150 n \beta^{6} r_{00} \alpha^{3}-108 b^{2} \beta^{7} r_{00} \alpha^{2}-72 \beta^{9} \alpha^{2}+36 \beta^{8} r_{0} \alpha^{2}-108 \beta^{8} r_{00} \alpha^{2}$ $+72 \beta^{9} r_{00} \alpha-63 n \beta^{8} r_{00} \alpha+27 n \beta^{9} r_{00}+18 \beta^{2} r_{00} \alpha^{7}+14 \beta^{3} r_{00} \alpha^{6}-76 \beta^{4} r_{00} \alpha^{5}+72 \beta^{8} r_{00} \alpha^{3}-18 \beta^{5} r_{00} \alpha^{4}+126 \beta^{6} r_{00} \alpha^{3}$ $-99 \beta^{8} r_{00} \alpha+90 \beta^{9} r_{00}-16 n \beta^{5} r_{00} \alpha^{5}+60 n \beta^{7} \alpha^{4}-48 b^{2} \beta^{3} r_{00} \alpha^{8}-8 b^{4} \beta r_{00} \alpha^{9}-16 n b^{4} \beta^{5} \alpha^{6}+32 n b^{4} \beta^{3} \alpha^{8}+60 n b^{2} \beta^{2} r_{00} \alpha^{7}$ $+12 b_{2} \beta^{2} r_{00} \alpha^{8}-4 n \beta^{3} \alpha^{8}-72 n b^{2} \beta^{6} \alpha^{5}-54 n \beta^{7} r_{00} \alpha^{3}+72 b^{2} \beta^{7} \alpha^{4}-10 \beta^{5} r_{00} \alpha^{6}+108 \beta^{8} \alpha^{3}-52 \beta^{4} r_{00} \alpha^{6}-12 \beta^{2} r_{0} \alpha^{8}$ $\left.-81 n \beta^{7} r_{00} \alpha^{2}-54 \beta^{7} r_{00} \alpha^{2}\right)$,

\footnotetext{
$W:=\tilde{W}\left(2 n b^{2} \alpha^{5}-4 n b^{2} \beta \alpha^{4}-2 n b^{2} \beta^{2} \alpha^{3}-6 \beta^{5}+2 n b^{2} \beta^{3} \alpha^{2}+2 b^{2} \alpha^{5}+n \alpha^{5}+2 b^{2} \beta \alpha^{4}-2 n \beta \alpha^{4}-2 b^{2} \beta^{2} \alpha^{3}-4 n \beta^{2} \alpha^{3}\right.$ $\left.-4 \beta^{2} \alpha^{3}+2 b^{2} \beta^{3} \alpha^{2}+7 n \beta^{3} \alpha^{2}+3 n \beta^{4} \alpha-3 n \beta^{5}+\alpha^{5}-2 \beta \alpha^{4}+4 \beta^{3} \alpha^{2}+3 \beta^{4} \alpha\right)$,
}

\footnotetext{
$b_{1}:=32 n b^{10} \beta \alpha^{15}-64 n b^{10} \beta^{2} \alpha^{14}-32 n b^{10} \beta^{3} \alpha^{13}+32 b^{10} \beta^{4} \alpha^{12}+32 b^{10} \beta \alpha^{15}+\beta \alpha^{13}+16 n b^{8} \beta \alpha^{15}+32 b^{10} \beta^{2} \alpha^{14}+32 n b^{8} \beta^{2} \alpha^{14}$ $-416 n b^{8} \beta^{3} \alpha^{13}+192 b^{6} \beta^{3} \alpha^{13}+32 b^{10} \beta^{4} \alpha^{12}+496 n b^{8} \beta^{4} \alpha^{12}+336 n b^{8} \beta^{5} \alpha^{11}-64 n b^{10} \beta \alpha^{13}+32 n b^{10} \alpha^{14}-224 b^{8} \beta^{3} \alpha^{13}$ $+32 b^{8} \beta^{2} \alpha^{14}-272 n b^{8} \beta^{6} \alpha^{10}-44 \beta^{6} \alpha^{10}-176 b^{2} \beta^{4} \alpha^{11}-134 n b^{2} \beta^{3} \alpha^{11}-96 \beta^{5} \alpha^{11}-40 b^{4} \beta^{3} \alpha^{11}-28 n \beta^{4} \alpha^{11}+64 n b^{2} \beta^{4} \alpha^{10}$ $-1028 b^{4} \beta^{4} \alpha^{10}+392 b^{2} \beta^{5} \alpha^{10}+94 n \beta^{5} \alpha^{10}+18 \beta^{4} \alpha^{12}-6 n \beta^{3} \alpha^{12}+632 b^{2} \beta^{6} \alpha^{9}+1046 b^{2} \beta^{5} \alpha^{9}+384 b^{4} \beta^{5} \alpha^{9}+\beta^{7} \alpha^{8}$ $-1224 b^{4} \beta^{7} \alpha^{7}-720 b^{2} \beta^{8} \alpha^{7}-3258 n b^{2} \beta^{7} \alpha^{7}-60 \beta^{8} \alpha^{8}+32 n b^{8} \beta^{2} \alpha^{13}+b^{2} \beta^{4} \alpha^{12}+128 n b^{6} \beta^{2} \alpha^{14}-24 n b^{6} \beta^{15}+64 n b^{10} \beta^{3} \alpha^{11}$ $+336 b^{8} \beta^{5} \alpha^{11}+32 n b^{8} \beta^{4} \alpha^{11}+1416 n b^{6} \beta^{5} \alpha^{11}-64 n b^{10} \beta^{4} \alpha^{10}-320 b^{8} \beta^{6} \alpha^{10}-128 n b^{8} \beta^{2} \alpha^{13}-24 n b^{6} \beta^{4} \alpha^{12}-96 n b^{8} \beta^{3} \alpha^{12}$ $-224 b^{8} \beta^{4} \alpha^{12}-20 n b^{4} \beta \alpha^{15}-112 n b^{8} \beta \alpha^{13}+64 n b^{6} \beta^{2} \alpha^{13}-32 n b^{8} \beta^{4} \alpha^{10}-16 b^{10} \beta^{4} \alpha^{10}+b^{8} \beta^{5} \alpha^{10}-168 b^{6} \beta^{4} \alpha^{12}+888 b^{6} \beta^{5} \alpha^{11}$ $-308 n b^{4} \beta^{4} \alpha^{12}-176 n b^{6} \beta^{4} \alpha^{11}-64 b^{8} \beta^{4} \alpha^{11}+432 n b^{4} \beta^{5} \alpha^{11}+176 n b^{8} \beta^{3} \alpha^{11}+64 b^{10} \beta^{3} \alpha^{11}-192 n b^{6} \beta^{3} \alpha^{12}+32 b^{8} \beta^{2} \alpha^{13}$ $-64 n b^{6} \beta \alpha^{13}+32 n b^{4} \beta^{3} \alpha^{13}-14 n b^{4} \beta^{3} \alpha^{12}-24 b^{8} \beta^{6} \alpha^{8}+360 b^{4} \beta^{5} \alpha^{11}-300 n b^{2} \beta^{5} \alpha^{11}+512 b^{8} \beta^{3} \alpha^{11}-320 n b^{6} \beta^{4} \alpha^{11}$ $-312 n b^{4} \beta^{4} \alpha^{11}-88 b^{8} \beta^{4} \alpha^{10}+224 b^{6} \beta^{5} \alpha^{10}-160 n b^{6} \beta^{4} \alpha^{10}-48 b^{4} \beta^{6} \alpha^{10}-1152 b^{4} \beta^{7} \alpha^{9}+240 n b^{4} \beta^{6} \alpha^{9}+756 n b^{2} \beta^{7} \alpha^{9}$ $+384 n b^{6} \beta^{6} \alpha^{8}+1176 n b^{4} \beta^{5} \alpha^{10}+260 n b^{2} \beta^{6} \alpha^{10}-176 b^{8} \beta^{5} \alpha^{9}+320 b^{6} \beta^{6} \alpha^{9}+728 n b^{6} \beta^{5} \alpha^{9}-2664 n b^{4} \beta^{7} \alpha^{8}+576 b^{4} \beta^{8} \alpha^{8}$ $-192 b^{2} \beta^{5} \alpha^{11}-164 n b^{2} \beta^{4} \alpha^{11}+1296 n b^{4} \beta^{9} \alpha^{6}+648 n b^{2} \beta^{10} \alpha^{6}-648 n b^{4} \beta^{10} \alpha^{5}-864 n b^{2} \beta^{9} \alpha^{7}-360 n b^{6} \beta^{8} \alpha^{6}-26 n b^{2} \beta^{12} \alpha^{4}$ $-4 b^{4} \beta \alpha^{15}+12 b^{6} \alpha^{14}+16 n b^{4} \alpha^{14}+4 b^{2} \beta^{2} \alpha^{14}-2 n \beta^{2} \alpha^{14}-64 b^{6} \beta \alpha^{13}+48 b^{4} \beta^{2} \alpha^{13}-8 n b^{4} \beta \alpha^{13}+44 b^{2} \beta^{3} \alpha^{13}+16 n b^{2} \beta^{2} \alpha^{13}$ $-1080 b^{4} \beta^{10} \alpha^{6}-102 n \beta^{5} \alpha^{11}-976 b 2^{3} \beta^{4} \alpha^{10}+528 b^{4} \beta^{5} \alpha^{10}+100 n b^{4} \beta^{4} \alpha^{10}+572 n b^{2} \beta^{5} \alpha^{10}-32 n \beta^{6} \alpha^{10}-736 b^{6} \beta^{5} \alpha^{9}$ $+452 n b^{2} \beta^{6} \alpha^{9}-288 n b^{4} \beta^{6} \alpha^{8}-2304 n b^{2} \beta^{7} \alpha^{8}+72 b^{2} \beta^{8} \alpha^{8}-156 n \beta^{8} \alpha^{8}-8 b^{8} \alpha^{14}+88 b^{4} \beta^{6} \alpha^{9}-16 b^{2} \beta^{6} \alpha^{10}+1680 n b^{4} \beta^{5} \alpha^{9}$ $+528 b^{6} \beta^{6} \alpha^{8}-1368 b^{4} \beta^{7} \alpha^{8}-14 n b^{2} \beta^{2} \alpha^{12}-408 n b^{6} \beta^{7} \alpha^{7}-288 b^{4} \beta^{8} \alpha^{7}-540 b^{2} \beta^{9} \alpha^{7}+72 n b^{2} \beta^{8} \alpha^{7}-1026 b^{2} \beta^{8} \alpha^{6}$ $+1296 b^{2} \beta^{12} \alpha^{3}-1512 n b 2 \beta^{11} \alpha^{3}-648 \beta^{13} \alpha^{3}+702 n \beta^{12} \alpha^{3}+n \beta \alpha^{13}+648 \beta^{14} \alpha^{2}+486 n b^{2} \beta^{12} \alpha^{2}+648 n \beta^{13} \alpha^{2}-324 n \beta^{14} \alpha$ $+4 b^{2} \beta \alpha^{13}+2 \beta^{2} \alpha^{13}+16 b^{2} \beta^{2} \alpha^{12}-6 \beta^{3} \alpha^{12}-10 b^{2} \beta^{3} \alpha^{11}+1260 \beta^{9} \alpha^{6}-28 \beta^{4} \alpha^{11}-24 \beta^{3} \alpha^{11}-308 b^{2} \beta^{4} \alpha^{10}+88 \beta^{5} \alpha^{10}$ $+205 n \beta^{5} \alpha^{9}+7 n \beta^{4} \alpha^{10}+740 b^{2} \beta^{5} \alpha^{9}+110 b^{2} \beta^{6} \alpha^{8}-252 \beta^{8} \alpha^{7}-840 n \beta^{7} \alpha^{7}+48 n b^{6} \beta^{5} \alpha^{10}+60 n b^{4} \beta^{6} \alpha^{10}-32 b^{8} \beta^{6} \alpha^{9}$ $-102 \alpha^{9} b^{6} \beta^{7} \alpha^{9}-304 n b^{6} \beta^{6} \alpha^{9}+1032 b^{6} \beta^{8} \alpha^{8}-1296 n b^{4} \beta^{7} \alpha^{9}+2 n b^{8} \beta^{6} \alpha^{8}-480 n b^{6} \beta^{7} \alpha^{8}+104 n b^{4} \beta^{8} \alpha^{8}+240 n b^{6} \beta^{8} \alpha^{7}$ $-540 n b^{4} \beta^{10} \alpha^{6}-20 b^{4} \beta \alpha^{15}-4 n b^{2} \beta \alpha^{15}+48 n b^{6} \alpha^{14}+40 b^{4} \beta^{2} \alpha^{14}+4 n b^{2} \beta^{2} \alpha^{14}-112 b^{8} \beta \alpha^{13}+414 n \beta^{8} \alpha^{6}+3240 b^{2} \beta^{9} \alpha^{5}$ $-18 \beta^{10} \alpha^{5}+1755 n$ beta ${ }^{9} \alpha^{5}-1332 b^{2} \beta^{10} \alpha^{4}-1674 \beta^{11} \alpha^{4}-2322 b^{2} \beta^{11} \alpha^{3}+648 \beta^{12} \alpha^{3}-1728 n \beta^{11} \alpha^{3}+2052 b^{2} \beta^{12} \alpha^{2}$ $-324 \beta^{13} \alpha^{2}+891 n \beta^{12} \alpha^{2}-648 \beta^{14} \alpha+567 n \beta^{13} \alpha-24 \beta^{3} \alpha^{11}-14 \beta^{4} \alpha^{10}+193 \beta^{5} \alpha^{9}-744 \beta^{7} \alpha^{7}+102 \beta^{6} \alpha^{8}-180 \beta^{8} \alpha^{6}$ $+891 \beta^{13} \alpha-252 \beta^{10} \alpha^{4}-810 \beta^{14}-1728 \beta^{11} \alpha^{3}+1026 \beta^{12} \alpha^{2},+4 n b^{8} \alpha^{14}+64 n b^{10} \beta^{2} \alpha^{12}-64 b^{10} \beta \alpha^{13}+1539 \beta^{9} \alpha^{5}$ $+1980 b^{4} \beta^{6} \alpha^{8}+16 b^{8} \beta \alpha^{15}-296 n b^{6} \beta^{3} \alpha^{13}-32 b^{10} \beta^{3} \alpha^{13}+108 b^{4} \beta^{8} \alpha^{7}+34 n b^{2} \beta^{11} \alpha^{5}-612 n b^{2} \beta^{8} \alpha^{8}-528 b^{6} \beta^{7} \alpha^{8}$ $-24 n b^{6} \beta^{3} \alpha^{11}+64 b^{6} \beta^{2} \alpha^{13}+2 b^{2} \alpha^{14}-3276 n b^{4} \beta^{7} \alpha^{7}+504 \beta^{7} \alpha^{9}+432 b^{2} \beta^{7} \alpha^{9}+972 \beta^{13} \alpha^{2}-918 \beta^{10} \alpha^{4}+76 n b^{4} \beta^{9} \alpha^{7}$ $-176 n b^{8} \beta^{5} \alpha^{9}+136 \beta^{6} \alpha^{9}$
} 
$b_{2}:=16 n b^{8} \alpha^{15}-384 n b^{6} \beta^{2} \alpha^{14}-22 b^{2} \beta^{2} \alpha^{12}+48 \beta^{3} \alpha^{12}-96 n \beta^{2} \alpha^{12}+1920 b^{2} \beta^{3} \alpha^{11}+324 b^{2} \beta^{4} \alpha^{10}-616 \beta^{5} \alpha^{10}+88 \beta^{4} \alpha^{11}$ $-12528 n b^{2} \beta^{5} \alpha^{9}-136 \beta^{6} \alpha^{9}-812 n \beta^{5} \alpha^{9}+4836 b^{2} \beta^{6} \alpha^{8}+570 n \beta^{4} \alpha^{10}+2376 \beta^{7} \alpha^{8}-1352 n \beta^{6} \alpha^{8}+2150 b^{2} \beta^{7} \alpha^{7}-600 \beta^{8} \alpha^{7}$ $-3672 \beta^{9} \alpha^{6}-2700 b^{2} \beta^{9} \alpha^{6}+264 b 2 \beta^{7} \alpha^{8}-11454 b^{2} \beta^{8} \alpha^{6}+128 n b^{6} \beta^{4} \alpha^{12}-192 n b^{6} \beta^{5} \alpha^{11}+128 b^{6} \beta \alpha^{15}-192 n b^{4} \beta^{2} \alpha^{14}$ $+96 n b^{4} \beta \alpha^{15}+64 n b^{6} \beta^{6} \alpha^{10}+128 b^{6} \beta^{4} \alpha^{12}+192 n b^{6} \beta^{3} \alpha^{12}+1056 n b^{4} \beta^{4} \alpha^{12}-64 n b^{6} \beta^{4} \alpha^{11}+1056 n b^{4} \beta^{5} \alpha^{11}-12 b^{6} \beta^{5} \alpha^{11}$ $-576 n b^{4} \beta^{3} \alpha^{13}+416 b^{6} \beta \alpha^{13}+960 n \beta^{5} \alpha^{11}+4 b^{4} \beta^{4} \alpha^{11}+1752 n b^{4} \beta^{3} \alpha^{11}+1440 b^{2} \beta^{5} \alpha^{11}+432 n b^{2} \beta^{4} \alpha^{11}-192 b^{2} \beta^{4} \alpha^{12}$ $-34 \beta^{4} \alpha^{12}-64 n b^{6} \beta^{2} \alpha^{13}-6 b^{6} \beta^{3} \alpha^{11}-96 b^{4} \beta^{2} \alpha^{13}+24 n b^{4} \beta \alpha^{13}-192 b^{2} \beta^{3} \alpha^{13}+32 n b^{6} \alpha^{15}-48 n b^{2} \beta^{2} \alpha^{13}-456 b^{6} \beta^{2} \alpha^{12}$ $-48 n \beta^{3} \alpha^{13}+192 b^{4} \beta^{3} \alpha^{12}-1200 n b^{4} \beta^{2} \alpha^{12}+160 b^{6} \beta^{6} \alpha^{10}-576 n b^{4} \beta^{7} \alpha^{9}-128 n b^{6} \beta^{5} \alpha^{10}+576 n b^{4} \beta^{8} \alpha^{8}-1632 n b^{4} \beta^{6} \alpha^{10}$ $+64 n b^{6} \beta^{6} \alpha^{9}+96 n b^{2} \beta^{2} \alpha^{14}+48 n b^{6} \alpha^{14}-144 b^{4} \beta^{2} \alpha^{14}+288 n b^{4} \beta^{3} \alpha^{12}-96 \alpha^{14} b^{4}-12 n b^{2} \beta^{4} \alpha^{12}-58 b^{4} \beta^{4} \alpha^{12}+12 b^{6} \beta^{4} \alpha^{11}$ $+288 b^{4} \beta^{5} \alpha^{11}+38 n b^{4} \beta^{4} \alpha^{11}+2928 n b^{2} \beta^{5} \alpha^{11}+1568 n b^{6} \beta^{3} \alpha^{11}+32 n b^{6} \beta \alpha^{13}-64 b^{6} \beta^{2} \alpha^{13}-192 b^{4} \beta^{3} \alpha^{13}-816 n b^{6} \beta^{2} \alpha^{12}$ $+1104 n b^{4} \beta^{4} \alpha^{10}+768 b^{2} \beta^{6} \alpha^{10}-1536 n b^{2} \beta^{5} \alpha^{10}+1656 b^{6} \beta^{4} \alpha^{10}+440 n \beta^{6} \alpha^{10}-832 b^{6} \beta^{5} \alpha^{9}-480 b^{4} \beta^{6} \alpha^{9}-724 n b^{4} \beta^{5} \alpha^{9}$ $-38 n \beta^{7} \alpha^{9}+16 n \beta^{8} \alpha^{8}+312 n b^{4} \beta^{7} \alpha^{7}-148 n b^{2} \beta^{8} \alpha^{7}+128 \beta^{6} \alpha^{10}-192 n b^{4} \beta^{8} \alpha^{6}+5832 n \beta^{9} \alpha^{7}-672 b^{4} \beta^{8} \alpha^{7}-2880 b^{2} \beta^{10} \alpha^{6}$ $-2592 n \beta^{11} \alpha^{5}-4104 n \beta^{10} \alpha^{6}+1728 n \beta^{12} \alpha^{4}+1008 n b^{2} \beta^{10} \alpha^{5}+36 n b^{2} \alpha^{14}+48 \beta^{2} \alpha^{14}+408 b^{4} \beta \alpha^{13}-48 b^{2} \beta^{2} \alpha^{13}+1056 \beta^{5} \alpha^{11}$ $+600 n b^{2} \beta^{5} \alpha^{10}-1488 b^{2} \beta^{5} \alpha^{10}+258 n b^{2} \beta^{4} \alpha^{10}-376 n \beta^{5} \alpha^{10}-528 b^{2} \beta^{6} \alpha^{9}-4872 n b^{2} \beta^{5} \alpha^{9}-39 \beta^{7} \alpha^{9}-376 \beta^{6} \alpha^{9}-72 b^{4} \beta^{6} \alpha^{8}$ $-432 n b^{2} \beta^{3} \alpha^{13}-576 b^{4} \beta^{5} \alpha^{10}+2688 n b^{4} \beta^{4} \alpha^{10}-368 b^{2} \beta^{7} \alpha^{9}-480 n b^{2} \beta^{6} \alpha^{9}+1944 b^{4} \beta^{4} \alpha^{10}-2508 n b^{2} \beta^{6} \alpha^{8}+1824 \beta^{8} \alpha^{8}$ $+8 n \beta^{3} \alpha^{13}+240 b^{6} \beta^{2} \alpha^{12}-72 b^{4} \beta^{3} \alpha^{12}-76 n b^{4} \beta^{2} \alpha^{12}-16 b^{2} \beta^{4} \alpha^{12}-48 n b^{2} \beta^{3} \alpha^{12}+18 n \beta^{4}-384 \alpha^{11} b^{4} \beta^{4} \alpha^{12}-22 n b^{4} \beta^{3} \alpha^{11}$ $-72 \beta^{3} \alpha^{13}-8 n \beta^{2} \alpha^{13}+192 b^{2} \beta^{3} \alpha^{12}-588 n b^{2} \beta^{2} \alpha^{12}+24 n \beta^{3} \alpha^{12}-272 \beta^{4} \alpha^{12}+228 n \beta^{3} \alpha^{12}+384 b^{2} \beta^{4} \alpha^{11}+648 n b^{2} \beta^{3} \alpha^{11}$ $-1368 b^{2} \beta^{9} \alpha^{5}+1872 \beta^{10} \alpha^{5}-4626 n \beta^{9} \alpha^{5}+1800 n \beta^{10} \alpha^{4}+95 b^{2} \beta^{10} \alpha^{4}+2376 \beta^{11} \alpha^{4}-1728 \beta^{12} \alpha^{3}+1890 n \beta^{11} \alpha^{3}+18 \beta^{2} \alpha^{12}$ $-576 \beta^{4} \alpha^{10}-4772 \beta^{5} \alpha^{9}+3748 \beta^{6} \alpha^{8}+14196 \beta^{7} \alpha^{7}+224 b^{3} \beta^{5} \alpha^{10}-384 n b^{4} \beta^{5} \alpha^{10}+720 n b^{4} \beta^{6} \alpha^{9}-14 n b^{4} \beta^{7} \alpha^{8}-128 b^{3} \beta^{5} \alpha^{10}$ $-192 b^{4} \beta^{7} \alpha^{9}+56 n b^{4} \beta^{6} \alpha^{9}-594 n b^{2} \beta^{7} \alpha^{9}-48 b^{4} \beta^{8} \alpha^{8}+960 n b^{4} \beta^{7} \alpha^{8}+486 n \beta^{8} \alpha^{8}-480 n b^{4} \beta^{8} \alpha^{7}+3024 n b^{2} \beta^{9} \alpha^{7}-20 n b^{2} \beta^{10} \alpha^{6}$ $+6744 b^{4} \beta^{7} \alpha^{7}-672 b^{2} \beta^{8} \alpha^{7}-17370 \beta^{9} \alpha^{5}+13194 \beta^{10} \alpha^{4}+8154 \beta^{11} \alpha^{3}-6588 \beta^{12} \alpha^{2}-126 n \beta^{12} \alpha^{2}+6 \alpha^{14}-2 \beta \alpha^{13}-104 n b^{6} \beta^{5} \alpha^{9}$ $+512 n b^{6} \beta^{6} \alpha^{8}-8 n \beta \alpha^{15}-120 b^{6} \alpha^{14}+72 n b^{4} \alpha^{14}+48 n \beta^{2} \alpha^{14}-10698 \beta^{8} \alpha^{6}+96 \alpha^{15} b^{4} \beta+304 n \beta^{7} \alpha^{7}+82 n \beta^{3} \alpha^{11}-192 b^{6} \beta^{3} \alpha^{13}$ $-12 n b^{6} \beta \alpha^{14}+144 n b^{2} \beta^{3} \alpha^{12}-8 \alpha^{15} \beta+56 \beta^{8} \alpha^{6}-96 b^{4} \beta^{2} \alpha^{13}+368 b^{2} \beta^{9} \alpha^{7}-206 n b^{2} \beta^{9} \alpha^{6}+12 n \beta^{4} \alpha^{11}+368 b^{2} \beta^{7} \alpha^{8}+152 \beta^{7} \alpha^{8}$ $+48 b^{6} \beta^{3} \alpha^{11}-74 b^{4} \beta^{2} \alpha^{12}+562 \beta^{3} \alpha^{11}+64 b^{6} \beta^{6} \alpha^{9}$

$b_{3}:=128 n b^{6} \beta \alpha^{15}-64 n b^{8} \beta \alpha^{14}+48 n b^{8} \beta^{3} \alpha^{12}-16 n b^{8} \beta^{4} \alpha^{11}-16 b^{8} \beta^{4} \alpha^{11}+16 n b^{8} \beta^{5} \alpha^{10}+64 n b^{6} \beta \alpha^{14}+256 n b^{6} \beta^{4} \alpha^{11}$ $-36 n b^{6} \beta^{2} \alpha^{13}-32 b^{8} \beta^{5} \alpha^{10}-4320 n b^{6} \beta^{5} \alpha^{10}+80 n b^{6} \beta^{6} \alpha^{9}-80 b^{6} \beta^{7} \alpha^{8}+16 b^{2} \beta \alpha^{14}-120 n b^{4} \alpha^{15}+16 b^{6} \beta \alpha^{14}+96 n b^{4} \beta \alpha^{14}$ $+640 n b^{8} \beta^{2} \alpha^{11}-1760 b^{6} \beta^{3} \alpha^{12}-16 n b^{6} \beta^{2} \alpha^{12}+64 b^{6} \beta^{4} \alpha^{11}-416 b^{6} \beta^{3} \alpha^{11}-4 n \beta \alpha^{14}+240 n b^{4} \beta^{4} \alpha^{11}-112 n b^{8} \beta^{3} \alpha^{10}$ $+32 n b^{6} \beta \alpha^{13}+56 n b^{6} \alpha^{13}+4 n b^{4} \beta \alpha^{13}-240 b^{4} \beta^{2} \alpha^{13}+14 n b^{4} \beta^{2} \alpha^{13}+48 n b^{4} \beta^{2} \alpha^{13}+6 n b^{2} \beta^{3} \alpha^{13}-24 b^{2} \beta^{2} \alpha^{13}+128 b^{6} \beta^{2} \alpha^{12}$ $-86 b^{4} \beta^{3} \alpha^{11}-16 n b^{2} \beta^{4} \alpha^{11}+68 b^{8} \beta^{3} \alpha^{10}+24 b^{4} \beta^{3} \alpha^{12}-24 b^{4} \beta^{2} \alpha^{12}+152 b^{6} \alpha^{13}-320 b^{4} \beta^{2} \alpha^{11}-24 b^{6} \beta^{3} \alpha^{11}+128 b^{6} \beta^{2} \alpha^{11}$ $-448 b^{8} \beta^{4} \alpha^{9}+52 b^{6} \beta^{5} \alpha^{9}+4952 n b^{6} \beta^{4} \alpha^{9}+48 b^{4} \beta^{6} \alpha^{9}+242 n b^{4} \beta^{5} \alpha^{9}-4 n \beta^{3} \alpha^{12}+1464 n b^{2} \beta^{6} \alpha^{9}-448 b^{6} \beta^{6} \alpha^{8}+24 n b^{6} \beta^{5} \alpha^{7}$ $-240 n b^{6} \beta^{7} \alpha^{7}+192 b^{6} \beta^{7} \alpha^{7}-2800 n b^{6} \beta^{6} \alpha^{7}+1152 b^{4} \beta^{7} \alpha^{8}+2160 n b^{4} \beta^{6} \alpha^{8}+384 b^{2} \beta^{8} \alpha^{8}+3984 n b^{2} \beta^{7} \alpha^{8}-192 b^{6} \beta^{4} \alpha^{10}$ $+1128 b^{4} \beta^{7} \alpha^{8}-4632 n b^{4} \beta^{6} \alpha^{8}-660 n b^{2} \beta^{7} \alpha^{8}+256 b^{8} \beta^{5} \alpha^{8}-396 b^{4} \beta^{8} \alpha^{7}+120 b^{4} \beta^{2} \alpha^{12}+3120 n b^{4} \beta^{7} \alpha^{7}-2124 n b^{2} \beta^{8} \alpha^{7}$ $+142 n b^{2} \beta^{9} \alpha^{6}-576 n b^{2} \beta^{11} \alpha^{4}+86 n b^{2} \beta^{10} \alpha^{5}-864 n b^{4} \beta^{9} \alpha^{5}-60 b^{2} \beta^{2} \alpha^{13}+24 n b^{2} \beta \alpha^{13}+8 b^{4} \beta \alpha^{13}+36 n b^{4} \alpha^{13}+24 n \beta^{2} \alpha^{13}$ $-672 n b^{4} \beta^{2} \alpha^{11}-176 b^{2} \beta^{4} \alpha^{11}+1496 b^{6} \beta^{2} \alpha^{11}+264 b^{2} \beta^{3} \alpha^{12}-12 n b^{2} \beta^{2} \alpha^{12}+384 n b^{4} \beta \alpha^{12}-504 n b^{2} \beta^{3} \alpha^{11}-196 n \beta^{4} \alpha^{11}$ $-7104 n b^{4} \beta^{3} \alpha^{10}-520 b^{2} \beta^{5} \alpha^{10}+600 n b^{2} \beta^{4} \alpha^{10}+228 n \beta^{5} \alpha^{10}-3256 b^{6} \beta^{4} \alpha^{9}+1392 b^{2} \beta^{6} \alpha^{9}+2700 n b^{2} \beta^{5} \alpha^{9}+532 n \beta^{6} \alpha^{9}$ $+18240 n b^{4} \beta^{5} \alpha^{8}-216 b^{2} \beta^{7} \alpha^{8}-4080 b^{2} \beta^{6} \alpha^{8}-2304 b^{4} \beta^{7} \alpha^{7}-34608 n b^{4} \beta^{6} \alpha^{7}+2016 b^{4} \beta^{5} \alpha^{9}+11160 n b^{4} \beta^{4} \alpha^{9}-880 \beta^{7} \alpha^{8}$ $+4480 n b^{6} \beta^{3} \alpha^{12}-480 b^{6} \beta^{2} \alpha^{13}-312 n b^{4} \beta^{2} \alpha^{13}-320 n b^{8} \beta \alpha^{12}+396 n b^{4} \beta^{3} \alpha^{12}-32 n b^{6} \beta \alpha^{12}+60 b^{4} \beta^{4} \alpha^{11}-9360 b^{4} \beta^{5} \alpha^{10}$ $-6240 b^{4} \beta^{3} \alpha^{11}+2400 n b^{4} \beta^{8} \alpha^{6}-316 n b^{2} \beta^{5} \alpha^{10}+24 n b^{2} \beta^{2} \alpha^{12}-5004 n b^{4} \beta^{6} \alpha^{8}-456 b^{4} \beta^{4} \alpha^{10}+2480 n b^{2} \beta \alpha^{12}-4 \beta^{3} \alpha^{12}$ $-2 n \beta^{2 \alpha^{12}}+1308 b^{4} \beta^{2} \alpha^{11}-456 b^{2} \beta^{3} \alpha^{11}-448 n b^{2} \beta^{2} \alpha^{11}-148 n \beta^{4} \alpha^{11}-600 b^{2} \beta^{4} \alpha^{10}-44840 n b^{2} \beta^{3} \alpha^{10}-100 n \beta^{3} \alpha^{11}$ $+108 \beta^{4} \alpha^{10}-122 b^{2} \beta^{6} \alpha^{10}+204 n b^{2} \beta^{5} \alpha^{8}-76 b^{4} \beta^{4} \alpha^{9}+2352 b^{2} \beta^{5} \alpha^{9}+72 n b^{2} \beta^{4} \alpha^{9}+34 \beta^{6} \alpha^{9}+70 n \beta^{5} \alpha^{9}+14688 b^{4} \beta^{5} \alpha^{8}$ $-4320 b^{2} \beta^{7} \alpha^{7}-3578 n b^{2} \beta^{6} \alpha^{7}-216 \beta^{8} \alpha^{7}-2298 n \beta^{7} \alpha^{7}-1459 b^{4} \beta^{7} \alpha^{6}+4908 b^{2} \beta^{8} \alpha^{6}-2658 n b^{2} \beta^{7} \alpha^{6}+564 \beta^{9} \alpha^{6}+3480 n \beta^{8} \alpha^{6}$ $+1296 \beta^{11} \alpha^{5}-26 n \beta^{10} \alpha^{5}-18 b^{4} \beta^{10} \alpha^{4}-2052 b^{2} \beta^{11} \alpha^{4}-1728 n b^{2} \beta^{10} \alpha^{4}-98 \beta^{12} \alpha^{4}-1728 n \beta^{11} \alpha^{4}-54 \beta^{11} \alpha^{4}-5562 n \beta^{10} \alpha^{4}$ $+612 n b^{2} \beta^{8} \alpha^{5}+2646 n \beta^{9} \alpha^{5}-123 b^{4} \beta^{9} \alpha^{4}-72 \beta^{10} \alpha^{5}-6732 b^{2} \beta^{10} \alpha^{4}-5382 n b^{2} \beta^{9} \alpha^{4}+108 \beta^{12} \alpha^{3}+41 \beta^{11} \alpha^{3}+7182 n b^{2} \beta^{11} \alpha^{2}$ $+4 \beta \alpha^{13}+308 b^{2} \beta-2 \beta^{2} \alpha^{12}+4 n \beta \alpha^{12}+10 b^{2} \alpha^{13}+80 b^{2} \beta^{2} \alpha^{11}+60 \beta^{4} \alpha^{10}-893 \beta^{3} \alpha^{10}-620 b^{2} \beta^{4} \alpha^{9}-87 n \beta^{6} \alpha^{8}-2160 b^{2} \beta^{7} \alpha^{7}$ $-428 \beta^{6} \alpha^{8}+5671 n \beta^{5} \alpha^{8}-9752 n \beta^{6} \alpha^{7}+18608 \alpha^{7} b 2 \beta^{5} \alpha^{7}-2424 \beta^{7} \alpha^{7}-30672 b^{2} \beta^{7} \alpha^{6}-100 \beta^{3} \alpha^{11}-80 n \alpha^{11} \beta^{2}-4148 b^{2} \beta^{3} \alpha^{10}$ $-352 n b^{8} \beta^{4} \alpha^{10}-16 b^{6} \beta^{6} \alpha^{9}-400 n b^{6} \beta^{6} \alpha^{8}-480 \beta^{7} \alpha^{8}-16 n b^{6} \beta^{5} \alpha^{9}+840 n b^{4} \beta^{6} \alpha^{9}+112 n b^{8} \beta^{5} \alpha^{8}+304 b^{6} \beta^{7} \alpha^{8}-108 n b^{4} \beta^{7} \alpha^{8}$ $-4 n b^{2} \alpha^{15}-12 b^{4} \alpha^{15}+48 b^{4} \beta \alpha^{14}+16 n b^{2} \beta \alpha^{14}+128 b^{8} \alpha^{13}-4770 \beta^{10} \alpha^{4}+121 n \beta^{9} \alpha^{4}+2208 \beta^{8} \alpha^{6}-14498 n \beta^{7} \alpha^{6}+37458 b^{2} \beta^{8} \alpha^{5}$ $-45648 b^{2} \beta^{10} \alpha^{3}+4212 \beta^{12} \alpha^{2}+3807 n \beta^{11} \alpha^{2}-1944 \beta^{13} \alpha+11583 n \beta^{12} \alpha-3429 n \beta^{13}+44 \beta \alpha^{12}-80 \beta^{2} \alpha^{11}-986 \beta^{3} \alpha^{10}+457 \beta^{4} \alpha^{9}$ $+15507 \beta^{8} \alpha^{5}+2016 \beta^{9} \alpha^{4}-31896 \beta^{10} \alpha^{3}-1170 \beta^{11} \alpha^{2}+2467 \beta^{12} \alpha-756 \beta^{11} \alpha^{3}-34200 n \beta^{10} \alpha^{3}+22932 b^{2} \beta^{11} \alpha^{2}-504 b^{2} \beta^{10} \alpha^{5}$ $+4158 n b^{2} \beta^{9} \alpha^{5}-932 n \beta^{6} \alpha^{8}-5196 b^{4} \beta^{6}-3224 \beta^{6} \alpha^{7}-17972 \beta^{7} \alpha^{6}+2815 n \beta^{8} \alpha^{5}+10188 b^{2} \beta^{9} \alpha^{4}-756 n b^{4} \beta^{8} \alpha^{7}+672 n b^{6} \beta^{4} \alpha^{10}$ $-90 n b^{4} \beta^{5} \alpha^{10}-756 n \beta^{13} \alpha+216 \beta^{13} \alpha^{2}+n \alpha^{13}+736 \beta^{5} \alpha^{9}+1477 n \beta^{4} \alpha^{9}-236 n b^{2} \beta^{10} \alpha^{3}+32 b^{2} \beta^{9} \alpha^{5}+516 n b^{4} \beta^{9} \alpha^{6}$ $+6442 \beta^{5} \alpha^{8}+3204 \beta^{9} \alpha^{5}-144 b^{6} \beta^{5} \alpha^{10}+14 n b^{6} \beta^{7} \alpha^{7}-8496 b^{2} \beta^{6} \alpha^{7}+68 n \beta^{12} \alpha^{2}+32 b^{8} \beta \alpha^{14}-4 b^{2} \alpha^{15}+32 b^{8} \alpha^{13}$ $+32 n b^{6} \beta \alpha^{13}-24 b^{8} \beta \alpha^{12}+8 n b^{2} \beta^{3} \alpha^{12}+288 b^{6} \beta^{8} \alpha^{7}+4448 b^{6} \beta^{5} \alpha^{8}+972 b^{4} \beta^{9} \alpha^{7}-5184 b^{4} \beta^{3} \alpha^{10}-352 \beta^{7} \alpha^{8} \alpha^{7}$ $+250 b^{4} \beta^{8} \alpha^{5}+3240 b^{2} \beta^{11} \alpha^{3}+1200 \beta^{5} \alpha^{10}-3064 b^{6} \beta^{6} \alpha^{7}-928 b^{6} \beta^{3} \alpha^{10}+16 b^{6} \beta \alpha^{12}-264 b^{4} \beta^{9} \alpha^{6}-3472 n b^{6} \beta^{3} \alpha^{10}$ 
$b_{4}:=16 b^{8} \alpha^{15}-16 n b^{8} \beta^{5} \alpha^{10}+80 n b^{8} \beta^{4} \alpha^{11}-48 n b^{8} \beta^{3} \alpha^{12}+96 n b^{8} \beta \alpha^{14}-48 n b^{4} \beta^{2} \alpha^{13}-24 b^{4} \alpha^{15}+520 b^{6} \beta^{5} \alpha^{10}-936 n b^{4} \beta^{3} \alpha^{12}$ $-32 n b^{6} \alpha^{15}+48 b^{8} \beta \alpha^{14}+192 n b^{6} \beta \alpha^{14}+64 b^{8} \beta^{2} \alpha^{13}-160 n b^{6} \beta^{2} \alpha^{13}-96 b^{8} \beta^{3} \alpha^{12}-672 n b^{6} \beta^{3} \alpha^{12}+80 b^{8} \beta^{4} \alpha^{11}+928 b^{6} \beta^{4} \alpha^{11}$ $+1704 n b^{4} \beta^{5} \alpha^{10}-576 b 2^{3} \beta^{6} \alpha^{9}+48 b^{4} \beta^{2} \alpha^{13}+48 n b^{2} \beta^{2} \alpha^{13}-4 b^{4} \beta^{3} \alpha^{12}-24 b^{4} \alpha^{15}+936 b^{2} \beta^{3} \alpha^{12}-432 b^{4} \beta^{4} \alpha^{11}-106 n b^{2} \beta^{4} \alpha^{11}$ $+120 b^{6} \beta^{7} \alpha^{8}-54 n b^{4} \beta^{7} \alpha^{8}+1080 n b^{4} \beta^{8} \alpha^{7}-2448 n b^{4} \beta^{6} \alpha^{9}+4 b^{4} \beta^{4} \alpha^{11}-32 b^{6} \alpha^{15}+400 n b^{2} \beta^{4} \alpha^{11}-720 b^{4} \beta^{3} \alpha^{12}-456 n b^{2} \beta^{3} \alpha^{12}$ $-864 b^{4} \beta^{6} \alpha^{9}+1512 b^{4} \beta^{8} \alpha^{7}+2808 n b^{2} \beta^{8} \alpha^{7}-1872 b^{2} \beta^{6} \alpha^{9}-1008 b^{4} \beta^{7} \alpha^{8}+81 \beta^{9} \alpha^{6}-1872 n b^{2} \beta^{7} \alpha^{8}+48 n b^{2} \beta \alpha^{14}+96 b^{4} \beta^{2} \alpha^{13}$ $-216 n b^{4} \beta^{9} \alpha^{6}-75 n \beta^{3} \alpha^{12}-198 b^{2} \beta^{7} \alpha^{8}-798 n \beta^{7} \alpha^{8}+1944 b^{2} \beta^{8} \alpha^{7}-384 n \beta^{6} \alpha^{9}-84 b^{2} \beta^{6} \alpha^{9}+3 n \beta^{5} \alpha^{10}+112 b^{2} \beta^{4} \alpha^{11}+47 n \beta^{4} \alpha^{11}$ $+756 n \beta^{9} \alpha^{6}+1324 b^{2} \beta^{5} \alpha^{10}-1728 b^{2} \beta^{10} \alpha^{5}+378 b^{2} \beta^{11} \alpha^{4}-1188 n \beta^{10} \alpha^{5}-72 \beta^{3} \alpha^{12}-135 n \beta^{11} \alpha^{4}+4 \beta^{2} \alpha^{13}-288 \beta^{6} \alpha^{9}+35 \beta^{4} \alpha^{11}$ $+837 \beta^{8} \alpha^{7}-480 n b^{6} \beta^{6} \alpha^{9}+96 n b^{6} \beta^{7} \alpha^{8}+120 b^{6} \beta \alpha^{14}+144 n b^{4} \beta \alpha^{14}+128 b^{6} \beta^{2} \alpha^{13}-1188 \beta^{10} \alpha^{5}-216 \beta^{11} \alpha^{4}+729 \beta^{12} \alpha^{3}-162 \beta^{13} \alpha^{2}$ $-1704 n b^{2} \beta^{5} \alpha^{10}+256 n b^{6} \beta^{5} \alpha^{10}+35 \beta^{4} \alpha^{11}-32 b^{6} \beta^{4} \alpha^{11}+1056 b^{4} \beta^{4} \alpha^{11}+108 b^{4} \beta \alpha^{14}+1504 n b^{2} \beta^{5} \alpha^{10}+1053 n \beta^{8} \alpha^{7}+405 n \beta^{12} \alpha^{3}$ $-768 \beta^{7} \alpha^{8}-50 b^{6} \beta^{3} \alpha^{12}-16 b^{8} \beta^{5} \alpha^{10}+720 b^{4} \beta^{5} \alpha^{10}+150 b^{4} \beta^{5} \alpha^{10}+80 n b^{2} \beta^{2} \alpha^{13}+8 b^{2} \beta^{9} \alpha^{6}+338 \beta^{5} \alpha^{10}-8 n b^{2} \alpha^{15}$

$b_{5}:=32 n b^{8} \beta \alpha^{14}+32 n b^{8} \beta^{3} \alpha^{12}+25 n b^{6} \beta^{4} \alpha^{12}+18 n b^{6} \beta^{5} \alpha^{11}-6 n b^{8} \beta^{2} \alpha^{13}+6 n b^{8} \beta^{4} \alpha^{11}-32 n b^{8} \beta^{5} \alpha^{10}-128 n b^{6} \beta^{3} \alpha^{13}$ $-32 b^{8} \beta^{5} \alpha^{10}-12 n b^{6} \beta^{3} \alpha^{12}+16 n b^{4} \beta^{5} \alpha^{11}-128 b^{3} \beta^{6} \alpha^{10}+2 b^{8} \beta \alpha^{11} \alpha^{14}+64 n b^{6} \beta \alpha^{14}-128 b^{6} \beta^{3} \alpha^{13}-12 n b^{6} \beta^{2} \alpha^{13}-12 n b^{4} \beta^{3} \alpha^{13}$ $-64 b^{8} \beta^{3} \alpha^{12}+74 n b^{6} \beta^{4} \alpha^{11}-48 b^{6} \beta^{2} \alpha^{13}-192 b^{4} \beta^{3} \alpha^{13}-144 n b^{4} \beta^{2} \alpha^{13}-272 b^{6} \beta^{3} \alpha^{12}-19 b^{4} \beta^{4} \alpha^{12}-24 b^{4} \beta^{3} \alpha^{12}-192 n b^{2} \beta^{4} \alpha^{12}$ $+38 b^{4} \beta^{5} \alpha^{11}-96 n b^{2} \beta^{3} \alpha^{13}+90 n b^{4} \beta^{4} \alpha^{11}+1440 n b^{2} \beta^{5} \alpha^{11}+22 b^{6} \beta^{5} \alpha^{10}-432 b^{6} \beta^{6} \alpha^{9}-960 b^{4} \beta^{7} \alpha^{9}-1872 n b^{4} \beta^{6} \alpha^{9}+720 n b^{4} \beta^{7} \alpha^{8}$ $+288 n b^{2} \beta^{6} \alpha^{10}-418 n b^{2} \beta^{7} \alpha^{9}+40 b^{6} \beta^{7} \alpha^{8}+960 b^{4} \beta^{8} \alpha^{8}+1152 n b^{2} \beta^{8} \alpha^{8}+84 n b^{4} \beta^{8} \alpha^{7}-42 n b^{4} \beta^{9} \alpha^{6}+216 n b^{2} \beta^{9} \alpha^{7}-864 n b^{2} \beta^{10} \alpha^{6}$ $-96 b^{2} \beta^{3} \alpha^{13}-48 n b^{2} \beta^{2} \alpha^{13}-72 b^{4} \beta^{2} \alpha^{13}+16 n b^{2} \beta \alpha^{14}+144 b^{4} \beta^{2} \alpha^{13}+352 \beta^{5} \alpha^{11}+600 b^{4} \beta^{5} \alpha^{10}+96 b^{2} \beta^{6} \alpha^{10}+272 n b^{2} \beta^{5} \alpha^{10}$ $+144 n b^{2} \beta^{7} \alpha^{8}-16 n \beta^{3} \alpha^{13}+1152 b^{2} \beta^{5} \alpha^{11}+46 n b^{2} \beta^{4} \alpha^{11}+456 b^{2} \beta^{4} \alpha^{11}-220 \beta^{3} \alpha^{12}-312 b^{4} \beta^{3} \alpha^{12}-128 n b^{2} \beta^{3} \alpha^{12}-96 b^{2} \beta^{4} \alpha^{12}$ $-1584 n b^{2} \beta^{6} \alpha^{9}-1824 n \beta^{7} \alpha^{9}-72 b^{4} \beta^{7} \alpha^{8}+1248 b^{2} \beta^{8} \alpha^{8}-432 n \beta^{8} \alpha^{8}+1080 b^{4} \beta^{8} \alpha^{7}+2160 n b^{2} \beta^{8} \alpha^{7}-16 \beta^{4} \alpha^{12}-220 n \beta^{3} \alpha^{12}$ $-256 n b^{6} \beta^{5} \alpha^{10}-960 n b^{4} \beta^{6} \alpha^{10}+320 b^{2} \beta^{4} \alpha^{11}+416 b^{2} \beta^{5} \alpha^{10}+32 \beta^{6} \alpha^{10}+82 n \beta^{5} \alpha^{10}-1080 b^{2} \beta^{6} \alpha^{9}-2256 \beta^{7} \alpha^{9}-360 b^{2} \beta^{7} \alpha^{8}$ $+360 \beta^{9} \alpha^{7}+864 n \beta^{8} \alpha^{7}-42 b^{2} \beta^{9} \alpha^{6}-1440 \beta^{10} \alpha^{6}-162 n \beta^{9} \alpha^{6}-118 b^{2} \beta^{10} \alpha^{5}-22 \beta^{11} \alpha^{5}-918 n \beta^{10} \alpha^{5}+76 b^{2} \beta^{11} \alpha^{4}+126 \beta^{12} \alpha^{4}$ $+70 \beta^{4} \alpha^{11}-162 n \beta^{13} \alpha^{2}-132 \beta^{7} \alpha^{8}-54 \beta^{9} \alpha^{6}+88 \beta^{5} \alpha^{10}-324 \beta^{6} \alpha^{9}-34 n b^{6} \beta^{4} \alpha^{10}+756 \beta^{8} \alpha^{7}+1104 b^{4} \beta^{6} \alpha^{10}-162 n b^{4} \beta^{5} \alpha^{10}$ $+576 n b^{4} \beta^{8} \alpha^{8}-960 n b^{4} \beta^{7} \alpha^{9}+192 n b^{6} \beta^{7} \alpha^{8}+64 b^{6} \beta \alpha^{14}+48 n b^{4} \beta \alpha^{14}-918 \beta^{10} \alpha^{5}+378 \beta^{11} \alpha^{4}+486 \beta^{12} \alpha^{3}-324 \beta^{13} \alpha^{2}$ $-2400 b^{2} \beta^{7} \alpha^{9}-6 \beta^{2} \alpha^{13}-108 b^{4} \beta^{6} \alpha^{9}-84 n \beta^{7} \alpha^{8}+128 b^{2} \beta^{8} \alpha^{7}+496 \beta^{5} \alpha^{11}+76 n \beta^{4} \alpha^{11}-384 n b^{6} \beta^{6} \alpha^{9}-128 n b^{6} \beta^{6} \alpha^{10}$ $+384 \beta^{8} \alpha^{8}+18 b^{6} \beta^{5} \alpha^{11}+2880 b^{2} \beta^{9} \alpha^{7}+2 \beta \alpha^{14}+324 n \beta^{12} \alpha^{3}-1680 n b^{2} \beta^{6} \alpha^{10}+38 n \beta^{11} \alpha^{4}-640 n \beta^{4} \alpha^{12}+368 n \beta^{6} \alpha^{10}$ $+48 b^{4} \beta \alpha^{14}+9 n b^{4} \beta^{5} \alpha^{10}+224 b^{6} \beta^{4} \alpha^{11}-28 b^{6} \beta^{4} \alpha^{12}$

$b_{6}:=256 n b^{6} \beta^{2} \alpha^{13}+96 n b^{6} \beta^{3} \alpha^{12}-160 n b^{6} \beta^{4} \alpha^{11}+64 n b^{8} \beta^{5} \alpha^{10}+104 b^{3} \beta^{6} \alpha^{10}-131 n b^{6} \beta^{6} \alpha^{10}-32 n b^{8} \beta^{6} \alpha^{9}-936 n b^{6} \beta^{7} \alpha^{9}$ $+32 b^{6} \beta^{5} \alpha^{10}+48 n b^{4} \alpha^{15}+96 b^{6} \beta \alpha^{14}-288 n b^{4} \beta \alpha^{14}-128 b^{6} \beta^{2} \alpha^{13}+108 n b^{4} \beta^{3} \alpha^{12}+240 b^{4} \beta^{2} \alpha^{13}-192 b^{6} \beta^{3} \alpha^{12}-160 b^{6} \beta^{4} \alpha^{11}$ $+504 n b^{2} \beta^{7} \alpha^{8}+2448 n b^{2} \beta^{6} \alpha^{9}+1104 b^{4} \beta^{6} \alpha^{9}+960 b^{2} \beta^{3} \alpha^{12}+228 n \beta^{3} \alpha^{12}+4 n \alpha^{15}-1248 b^{2} \beta^{4} \alpha^{11}-200 n \beta^{4} \alpha^{11}-86 b^{2} \beta^{5} \alpha^{10}$ $-768 b^{2} \beta^{7} \alpha^{8}+936 n \beta^{7} \alpha^{8}-2232 b^{2} \beta^{8} \alpha^{7}+216 n b^{2} \beta^{9} \alpha^{6}-216 b^{2} \beta \alpha^{14}-1080 n b^{2} \beta^{8} \alpha^{7}-24 n \beta \alpha^{14}+144 b^{2} \beta^{2} \alpha^{13}-4 n \beta^{2} \alpha^{13}$ $-1080 \beta^{9} \alpha^{7}+1944 b^{4} \beta^{9} \alpha^{5}+24 b^{2} \beta^{9} \alpha^{6}+486 \beta^{10} \alpha^{6}+1296 n b^{2} \beta^{8} \alpha^{6}+1458 n \beta^{9} \alpha^{6}-242 \beta^{8} \alpha^{7}+10 \beta^{9} \alpha^{6}-1208 \beta^{5} \alpha^{10}$ $+10 \beta^{7} \alpha^{8}+14 \beta^{10} \alpha^{5}+24 b^{2} \alpha^{15}-50 \beta^{11} \alpha^{4}-14 b^{4} \beta \alpha^{14}+48 n b^{4} \alpha^{15}+32 b^{6} \alpha^{15}-92 \beta^{7} \alpha^{8}+24 b^{2} \alpha^{15}-75 \beta^{5} \alpha^{10}$ $+468 \beta^{3} \alpha^{12}-56 \beta^{4} \alpha^{11}-18 \beta^{8} \alpha^{7}-54 b^{4} \beta^{8} \alpha^{6}+25 b^{2} \beta^{6} \alpha^{9}+66 n b^{6} \beta^{8} \alpha^{8}-24 b^{6} \beta \alpha^{15}+96 n \beta^{6} \alpha^{9}-132 n b^{4} \beta^{4} \alpha^{11}$ $+1752 \beta^{6} \alpha^{9}-14 n \alpha^{15} b^{2} \beta \alpha^{15}$

$b_{7}:=48 b^{6} \beta^{3} \alpha^{12}+152 b^{8} \beta^{2} \alpha^{12}-120 n b^{6} \beta^{2} \alpha^{12}-128 b^{4} \beta^{4} \alpha^{12}-52 n+816 b^{6} \beta^{3} \alpha^{12}+528 b^{6} \beta^{7} \alpha^{8}-1200 b^{6} \beta^{5} \alpha^{10}-96 b^{8} \beta \alpha^{14}$ $+72 b^{4} \beta^{3} \alpha^{12}-24 b^{4} \beta^{5} \alpha^{10}+26 b^{4} \beta^{7} \alpha^{8}-12 \beta^{5} \alpha^{10}-18 b^{2} \beta^{5} \alpha^{10}+52 \beta^{7} \alpha^{8}+12 \beta^{3} \alpha^{12}+972 b^{2} \beta^{11} \alpha^{4}-10 \beta^{9} \alpha^{6}-72 b^{4} \beta \alpha^{14}$ $+192 b^{8} \beta^{3} \alpha^{12}-96 b^{8} \beta^{5} \alpha^{10}-4 b^{6} \beta \alpha^{14}+972 \beta^{11} \alpha^{4}$,

$b_{8}:=96 b^{6} \beta^{2} \alpha^{13}+128 b^{6} \beta^{3} \alpha^{12}-192 n b^{4} \beta^{3} \alpha^{12}-128 b^{6} \beta^{4} \alpha^{11}-1008 n b^{4} \beta^{4} \alpha^{11}+1008 n b^{4} \beta^{6} \alpha^{9}+32 b^{6} \beta^{6} \alpha^{9}-128 b^{6} \beta^{7} \alpha^{8}$ $+1104 n b^{4} \beta^{5} \alpha^{10}-256 n b^{6} \beta^{3} \alpha^{12}-128 n b^{6} \beta^{4} \alpha^{11}+96 n b^{6} \beta^{2} \alpha^{13}+48 n b^{4} \beta^{9} \alpha^{6}+72 n b^{2} \beta^{2} \alpha^{13}-288 b^{4} \beta^{4} \alpha^{11}$ $-1800 n b^{2} \beta^{7} \alpha^{8}-288 b^{4} \beta^{8} \alpha^{7}-1296 n b^{2} \beta^{8} \alpha^{7}-5304 b^{4} \beta^{5} \alpha^{9}+432 b^{4} \beta^{6} \alpha^{9}-48 \beta^{5} \alpha^{10}+2208 b^{2} \beta^{6} \alpha^{9}-672 n b^{4} \beta^{7} \alpha^{8}$ $+60 n \beta^{5} \alpha^{10}+1416 \alpha^{9} b^{2} \beta^{6} \alpha^{9}+856 n \beta^{6} \alpha^{9}-576 b^{2} \beta^{7} \alpha^{8}-664 n \beta^{7} \alpha^{8}-1656 b^{2} \beta^{8} \alpha^{7}-936 n n \beta^{8} \alpha^{7}+1536 b^{2} \beta^{9} \alpha^{6}$ $-504 n \beta^{11} \alpha^{4}+324 n \beta^{12} \alpha^{3}-108 n \beta^{13} \alpha^{2}-220 \beta^{4} \alpha^{11}+496 \beta^{6} \alpha^{9}-432 \beta^{8} \alpha^{7}-136 \beta^{7} \alpha^{8}+600 \beta^{9} \alpha^{6}+288 n b^{6} \beta^{5} \alpha^{10}$ $-648 \beta^{11} \alpha^{4}+36 \beta^{10} \alpha^{5}+108 \beta^{12} \alpha^{3}-192 b^{2} \beta^{5} \alpha^{10}-220 n \beta^{4} \alpha^{11}-192 b^{4} \beta^{5} \alpha^{10}+16 \beta^{3} \alpha^{12}+12 \beta^{2} \alpha^{13}-36 n \beta^{10} \alpha^{5}$ $+16 n \beta^{3} \alpha^{12}-552 b^{2} \beta^{4} \alpha^{11}-960 n b^{4} \beta^{7} \alpha^{8}-144 n b^{4} \beta^{8} \alpha^{7}-912 n b^{2} \beta^{4} \alpha^{11}+192 b^{4} \beta^{3} \alpha^{12}+1200 n \beta^{9} \alpha^{6}+720 b^{2} \beta^{10} \alpha^{5}$ $-864 b^{2} \beta^{11} \alpha^{4}+216 \beta^{13} \alpha^{2}+32 n b^{6} \beta^{6} \alpha^{9}-32 b^{6} \beta^{7} \alpha^{8}$,

$A_{16}:=4(n+1)\left(1+2 b^{2}\right)^{2}\left(6 b^{2} \beta+2 b^{2}+1\right) r_{00}^{2}$,

$A_{15}:=-\left(1+2 b^{2}\right)\left(16 n b^{8} \beta r_{000}-32 b^{6} \beta^{2} r_{00}-32 n b^{6} \beta^{2} r_{00}^{2}-32 n b^{6} \beta^{2} r_{00}+16 b^{6} \beta^{2} r_{00}^{2}-2 n b^{6} \beta r_{0} r_{00}+4 n b^{4} \beta^{2} r_{00}^{2}+16 b^{8} \beta r_{000}\right.$ $+16 n b^{4} \beta^{2} r_{00}+104 n b^{4} \beta r_{0} r_{00}+24 \beta^{2} r_{00}^{2}-8 n b^{6} b^{i} r_{i 00}-16 r_{00}^{2} b^{6}+16 b^{4} \beta^{2} r_{00}-12 n b^{4} \beta r_{000}-4 n b^{2} \beta r_{000}+16 b^{2} \beta^{2} r_{00}$ $+24 b^{2} \beta^{2} r_{00}^{2}+44 b^{2} \beta r_{0} r_{00}-4 n \beta^{2} r_{00}^{2}-8 b^{6} b^{i} r_{i 00}-12 b^{2} \beta r_{000}-12 n b^{4} b^{i} r_{i 00}+24 r_{0}^{2} b^{4}+8 r_{00} r_{0} b^{4}-16 r_{00}^{2} b^{4}+12 r_{0}^{2}$ $+2 n r_{0}^{2} b^{2}+8 n r_{00} r_{0} b^{2}-4 n b^{2} r_{00}^{2}-4 \beta^{2} r_{00}^{2}-8 n \beta r_{0} r_{00}-2 b^{4} b^{i} r_{i 00}-4 b^{2} \beta r_{000}-6 n b^{2} b^{i} r_{i 00}+4 r_{0}^{2} b^{2}+8 r_{00} r_{0} b^{2}-4 r_{00}^{2} b^{2}$ $+8 n r_{00} r_{0} b^{4}-4 n r_{00}^{2} b^{4}-32 b^{6} \beta r_{0} r_{00}+8 n r_{00}^{2} b^{6}-6 b^{2} b^{i} r_{i 00}-n b^{i} r_{i 00}+2 r_{00} r_{0}+16 n b^{2} \beta^{2} r_{00}+44 b^{2} \beta r_{0} r_{00}-8 n \beta r_{0} r_{00}$ $\left.+14 b^{4} \beta r_{0} r_{00}+4 n r_{0}^{2} b^{4}+2 n r_{00} r_{0}-b^{i} r_{i 00} r\right)$, 
$A_{14}:=2\left(1+2 b^{2}\right)\left(8 b^{6} \beta^{3} r_{00}^{2}+16 n b^{8} \beta^{2} r_{000}-32 n b^{6} \beta^{3} r_{00}+8 b^{6} \beta^{3} r_{00}^{2}-32 n b^{6} \beta^{2} r_{0} r_{00}+108 n b^{4} \beta^{3} r_{00}^{2}-8 b^{8} \beta^{2} r_{000}+16 b^{6} \beta^{3} r_{00}\right.$ $+72 n b^{4} \beta^{3} r_{00}+6 b^{4} \beta^{3} r_{00}^{2}+216 n b^{4} \beta^{2} r_{0} r_{00}-8 n b^{4} \beta^{2} r_{00}^{2}-36 n b^{2} \beta^{3} r_{00}^{2}-4 b^{6} \beta^{2} r_{000}-24 n b^{6} \beta b^{i} r_{i 00}-8 n b^{3} \beta r_{0}+16 b^{6} \beta r_{00}$ $-24 n b^{4} \beta^{2} r_{000}-6 b^{4} \beta^{2} r_{0} r_{00}-8 b^{4} \beta^{2} r_{00}^{2}+72 n b^{4} \beta r_{0}^{2}+24 n b^{4} \beta r_{0} r_{00}-8 b^{4} \beta r_{00}^{2}-24 b^{2} \beta^{3} r_{00}^{2}+60 n b^{2} \beta^{2} r_{0} r_{00}-8 n b^{2} \beta^{2} r_{00}^{2}$ $-8 b^{6} \beta r_{00}-12 n b^{6} r_{000}-8 b^{6} r_{0} r_{00}-b^{4} \beta^{2} r_{000}-36 n b^{4} \beta b^{i} r_{i 00}-12 n b^{4} \beta r_{0}-36 b^{4} \beta r_{0}^{2}-12 b^{4} \beta r_{0} r_{00}-b^{4} \beta r_{00}^{2}-4 b^{2} \beta^{2} r_{000}$ $+24 n b^{2} \beta r_{0} r_{00}-38 n b^{2} \beta r_{00}^{2}-4 n \beta^{3} r_{00}-8 \beta^{3} r_{00}^{2}-8 n \beta^{2} r_{0} r_{00}-2 n \beta^{2} r_{00}^{4}+4 b^{4} \beta r_{00}-6 n b^{4} r_{000}+30 b^{2} r_{0} r_{00}-4 b^{2} \beta^{2} r_{000}$ $+6 b^{2} \beta r_{0} r_{00}-8 b^{2} \beta r_{00}^{2}-2 b^{2} r_{0} r_{00}-4 \beta^{3} r_{00}+n \beta^{2} r_{00}-8 \beta^{2} r_{0} r_{00}-2 \beta^{2} r_{00}^{2}+6 \beta r_{0} r_{00}+2 n \beta r_{00}^{2}-3 b^{4} r_{000}-9 b^{2} \beta b^{i} r_{i 00}$ $-\beta r_{0}+72 n b^{2} \beta r_{0}^{2}+\beta^{2} r_{000}-3 n \beta b^{i} r_{i 00}+36 \beta r_{0}^{2}+6 \beta r_{0} r_{00}+2 \beta r_{00}^{2}-4 n r_{0} r_{00}-18 n b^{2} \beta r_{0} b^{i} r_{i 00}-3 \beta b^{i} r_{00}+24 b^{4} \beta^{3} r_{00}$ $+9 b^{2} r_{0}-12 b^{4} \beta r_{0}+8 n b^{6} \beta r_{00}^{2}-8 n b^{8} r_{000}+16 n b^{6} r_{0} r_{00}+16 b^{6} \beta^{2} r_{0} r_{00}-8 b^{2} \beta^{2} r_{00}^{2}+12 b^{6} \beta b^{i} r_{i 00}-n b^{2} r_{000}-b^{2} r_{000} r_{00}$ $-6 b^{2} \beta r_{0}+b^{6} \beta r_{00}^{2}-16 n b^{6} \beta^{2} r_{000}+4 b^{8} r_{000}-4 r_{0} r_{00}-n \beta r_{0}+16 n b^{4} \beta r_{00}+18 b^{2} \beta^{2} r_{0} r_{00}+54 b^{2} \beta r_{0}^{2}+4 b^{2} \beta r_{00}-8 n \beta^{3} r_{00}^{2}$ $\left.+4 n b^{2} \beta r_{00}-8 b^{6} \beta r_{0}\right)$,

$A_{13}:=-32 n b^{8} \beta^{4} r_{00}^{2}+320 n b^{10} \beta^{3} r_{000}-640 n b^{8} \beta^{4} r_{00}-32 b^{8} \beta^{4} r_{00}^{2}-64 n b^{8} \beta^{3} r_{0} r_{00}-768 n b^{6} \beta^{4} r_{00}^{2}+32 b^{10} \beta^{3} r_{000}-64 b^{8} \beta^{4} r_{00}$ $-160 n b^{8} \beta^{2} r_{00}^{2}-896 n b^{6} \beta^{4} r_{00}+96 b^{6} \beta^{4} r_{00}^{2}-1280 n b^{6} \beta^{3} r_{0} r_{00}+96 n b^{6} \beta^{3} r_{00}^{2}+504 n b^{4} \beta^{4} r_{00}^{2}+64 n b^{10} \beta r_{000}+224 b^{8} \beta^{3} r_{000}$ $-128 n b^{4} \beta^{2} r_{00}-32 n b^{8} \beta^{2} r_{000}-160 n b^{8} \beta^{2} r_{00}^{2}-128 n b^{8} \beta r_{0} r_{00}-224 b^{6} \beta^{4} r_{00}+128 n b^{6} \beta^{3} r_{0}+64 n b^{6} \beta^{3} r_{00}+64 b^{6} \beta^{3} r_{0} r_{00}$ $+1120 n b^{6} \beta^{2} r_{00}^{2}+48 n b^{2} \beta^{4} r_{00}+144 b^{4} \beta^{4} r_{00}^{2}+144 n b^{4} \beta^{3} r_{0} r_{00}+144 n b^{4} \beta^{3} r_{00}^{2}+64 b^{10} \beta r_{000}-64 b^{8} \beta^{2} b^{i} r_{i 00}-128 b^{8} \beta^{2} r_{00}$ $+112 n b^{8} \beta r_{000}-128 b^{8} \beta r_{0} r_{00}+64 n r_{00}^{2} b^{8}+128 b^{6} \beta^{3} r_{0}+64 b^{6} \beta^{3} r 00+\beta^{3} r_{00}^{2}+152 b^{6} \beta^{3} r_{000}+160 n b^{6} \beta^{2} b^{i} r_{i 00}+192 n b^{6} \beta^{2} r_{0}$ $+192 b^{6} \beta^{2} r_{0}^{2}+192 b^{6} \beta^{2} r_{0} r_{00}-272 b^{6} \beta^{2} r_{00}^{2}-80 n b^{6} \beta r_{0} r_{00}-32 n b^{6} \beta r_{00}^{2}-144 b^{4} \beta^{4} r_{00}+192 n b^{4} \beta^{3} r_{0}+96 n b^{4} \beta^{3} r_{00}$ $+144 b^{4} \beta^{3} r_{0} r_{00}+416 n b^{8} \beta^{3} r_{000}-64 n b^{6} \beta^{2} r_{000}+34 n b^{2} \beta^{4} r_{00}^{2}+26 n b^{6} \beta^{3} r_{000}+12 b^{8} \beta^{2} b^{i} r_{i 00}+48 b^{4} \beta^{3} r_{00}^{2}-360 n b^{4} \beta^{2} r_{0}^{2}$ $+114 n b^{4} \beta^{2} r_{00}^{2}+26 n b^{2} \beta^{4} r_{00}+22 b^{2} \beta^{4} r_{00}^{2}+58 n b^{2} \beta^{3} r_{0} r_{00}+72 n b^{2} \beta^{3} r_{00}^{2}-4 n \beta^{4} r_{00}^{2}+112 b^{8} \beta r_{000}-176 b^{8} r_{00}^{2}-128 b^{6} \beta^{2} b^{i} r_{i 00}$ $-36 b^{6} \beta^{2} r_{00}-6 b^{6} \beta^{2} r_{000}-48 \beta^{4} r_{00}^{2}+6 n b^{6} \beta r_{000}-68 b^{6} \beta r_{0} r_{00}-32 b^{6} \beta r_{00}^{2}+8 n r_{00}^{2} b^{6}+192 b^{4} \beta^{3} r_{0}+48 b^{2} \beta^{3} r_{00}^{2}+96 b^{4} \beta^{3} r_{00}$ $+48 b^{4} \beta^{2} b^{i} r_{i 00}+144 n b^{4} \beta^{2} r_{0}-144 n b^{4} \beta^{2} r_{00}+6 \beta^{2} r_{0}-48 n b^{4} \beta^{2} r_{000}+24 b^{4} \beta^{2} r_{0}^{2}+264 b^{4} \beta^{2} r_{0} r_{00}+432 b^{4} \beta^{2} r_{00}^{2}+96 n b^{4} \beta r_{0} r_{00}$ $-48 n b^{4} \beta r_{00}^{2}+28 b^{2} \beta^{4} r_{00}+96 n b^{2} \beta^{3} r_{0}+48 n b^{2} \beta^{3} r_{00}-56 n b^{2} \beta^{3} r_{000}+16 \beta^{3} r_{0}+28 b^{2} \beta^{3} r_{0} r_{00}-72 n b^{2} \beta^{2} r_{0}^{2}+72 n b^{2} \beta^{2} r_{0} r_{00}$ $+92 n \beta^{3} r_{0} r_{00}+12 n \beta^{3} r_{00}^{2}+64 b^{6} \beta r_{000}-152 r_{00}^{2} b^{6}-96 b^{4} \beta^{2} b^{i} r_{i 00}+72 b^{4} \beta^{2} r_{0}-216 b^{4} \beta^{2} r_{00}-48 b^{4} \beta^{2} r_{000}+8 n b^{4} \beta r_{000}$ $+96 b^{2} \beta^{3} r_{0}+48 b^{2} \beta^{3} r_{00}-4 b^{2} \beta^{3} r_{000}-8 n b^{2} \beta^{2} b^{i} r_{i 00}+48 n b^{2} \beta^{2} r_{0}-64 n b^{2} \beta^{2} r_{00}-16 n b^{2} \beta^{2} r_{000}-120 b^{2} \beta^{2} r_{0}^{2}+12 b^{2} \beta^{2} r_{0} r_{00}$ $-48 b^{4} \beta r_{00}^{2}+36 n r_{00}^{2} b^{4}+92 n b^{2} \beta r_{0} r_{00}-24 n b^{2} \beta r_{00}^{2}+8 \beta^{4} r_{00}+16 n \beta^{3} r_{0}+8 n \beta^{3} r_{00}-8 n \beta^{3} r_{000}+116 \beta^{3} r_{0} r_{00}+18 n \beta^{2} r_{0} r_{00}$ $+8 b^{4} \beta r_{000}-r_{00}^{2}-24 r_{00}^{2} b^{4}-2 b^{2} \beta^{2} b^{i} r_{i 00}+36 b^{2} \beta^{2} r_{0}-6 b^{2} \beta^{2} r_{00}-16 b^{2} \beta^{2} r_{000}-4 n b^{2} \beta r_{000}-4 b^{2} \beta r_{0} r_{00}-4 b^{2} \beta r_{00}^{2}+n b^{2} r_{00}^{2}$ $-8 \beta^{3} r_{000}-4 n \beta^{2} b^{i} r_{i 00}+6 n \beta^{2} r_{0}-12 n \beta^{2} r_{00}-2 n \beta^{2} r_{000}-42 \beta^{2} r_{0}^{2}+18 \beta^{2} r_{0} r_{00}-52 \beta^{2} r_{00}^{2}+20 n \beta r_{0} r_{00}-4 \beta r_{00}^{2}-4 b^{2} \beta r_{000}$ $-2 \beta^{2} r_{000}-n \beta r_{000}+20 \beta r_{0} r_{00}-4 \beta r_{00}^{2}-n r 00^{2}-\beta r_{000}+8 \beta^{3} r_{00}+6 n \beta^{2} r_{0}^{2}-4 \beta^{2} b^{i} r_{i 00}-32 b^{8} \beta^{2} r_{000}-64 b^{8} \beta^{3} r_{0} r_{00}$ $+16 b^{2} \beta^{2} r_{00}^{2}+14 n b^{2} \beta^{2} r_{00}^{2}+96 n b^{8} \beta^{2} r_{0}+2 r_{00}^{2} b^{2}-12 \beta^{2} r_{00}-384 n b^{6} \beta^{2} r_{0}^{2}-192 n b^{6} \beta^{2} r_{00}-52 n \beta^{2} r_{00}^{2}-32 n b^{4} \beta^{3} r_{000}$ $+72 n b^{4} \beta^{2} r_{0} r_{00}+48 b^{6} \beta^{2} r_{0}-432 b^{4} \beta r_{0} r_{00}-32 b^{4} \beta^{3} r_{000}+8 n \beta^{4} r_{00}$,

$A_{12}:=-\beta\left(32 n b^{10} \beta^{3} r_{000}-64 n b^{8} \beta^{4} r_{00}-64 n b^{8} \beta^{3} r_{0} r_{00}+32 b^{10} \beta^{3} r_{000}-64 b^{8} \beta^{4} r_{00}+496 n b^{8} \beta^{3} r_{000}-64 b^{8} \beta^{3} r_{0} r_{00}-28 n b^{8} \beta^{2} r_{00}^{2}\right.$ $-49 n b^{6} \beta^{3} r_{0} r_{00}+32 n b^{6} \beta^{3} r_{00}^{2}+2184 n b^{4} \beta^{4} r_{00}^{2}+64 n b^{10} \beta r_{000}-224 b^{8} \beta^{3} r_{000}-48 n b^{8} \beta^{2} b^{i} r_{i 00}+32 n b^{8} \beta^{2} r_{0}-128 n b^{8} \beta^{2} r_{00}$ $-128 n b^{8} \beta r_{0} r_{00}+480 b^{6} \beta^{4} r_{00}+256 n b^{6} \beta^{3} r_{0}+192 n b^{6} \beta^{3} r_{00}-24 n b^{6} \beta^{3} r_{000}+224 b^{6} \beta^{3} r_{0} r_{00}-64 b^{6} \beta^{3} r_{00}^{2}+144 n b^{6} \beta^{2} r_{0}^{2}$ $+1104 n b^{4} \beta^{4} r_{00}+792 b^{4} \beta^{4} r_{00}^{2}+2592 n b^{4} \beta^{3} r_{0} r_{00}-240 n b^{4} \beta^{3} r_{00}^{2}+168 n b^{2} \beta^{4} r_{00}^{2}-320 b^{10} \beta r_{000}+96 b^{8} \beta^{2} b^{i} r_{i 00}-604 b^{8} \beta^{2} r_{0}$ $+32 n r_{00}^{2} b^{8}-128 b^{6} \beta^{3} r_{0}-168 b^{6} \beta^{3} r_{000}-672 n b^{6} \beta^{2} b^{i} r_{i 00}-128 n b^{6} \beta^{2} r_{0}-256 n b^{6} \beta^{2} r_{00}-192 n b^{6} \beta^{2} r_{000}-288 b^{6} \beta^{2} r_{0}^{2}$ $-592 n b^{3} \beta r_{0} r_{00}-112 n b^{6} \beta r_{00}^{2}+384 b^{4} \beta^{4} r_{00}+192 n b^{4} \beta^{3} r_{00}-308 n b^{4} \beta^{3} r_{000}-72 b^{4} \beta^{3} r_{0} r_{00}-336 b^{4} \beta^{3} r_{00}^{2}+112 n b^{4} \beta^{2} r_{0}^{2}$ $+208 n b^{2} \beta^{4} r_{00}+42 b^{2} \beta^{4} r_{00}^{2}+72 n b^{2} \beta^{3} r_{0} r_{00}-24 n b^{2} \beta^{3} r_{00}^{2}-30 n \beta^{4} r_{00}^{2}+152 b^{8} \beta r_{000}-12 r_{00}^{2} b^{8}+312 b^{6} \beta^{2} b^{i} r_{i 00}-272 b^{6} \beta^{2} r_{0}$ $-12 n b^{6} \beta r_{000}-856 b^{6} \beta r_{0} r_{00}+32 b^{6} \beta r_{00}^{2}+48 n r_{00}^{2} b^{6}-12 b^{4} \beta^{3} r_{0}-14 n \beta^{4} r_{00}-96 n b^{4} \beta^{2} b^{i} r_{i 00}-20 n b^{4} \beta^{2} r_{0}-12 n b^{4} \beta^{2} r_{00}$ $+96 b^{4} \beta^{2} r_{0} r_{00}+744 b^{4} \beta^{2} r_{00}^{2}-840 n b^{4} \beta r_{0} r_{00}-168 n b^{4} \beta r_{00}^{2}+208 b^{2} \beta^{4} r_{00}-192 n b^{2} \beta^{3} r_{0}+48 n b^{2} \beta^{3} r_{00}-52 n b^{2} \beta^{3} r_{000}$ $-28 b^{2} \beta^{3} r_{00}^{2}-240 b^{4} \beta^{2} r_{0}^{2}+86 n b^{4} \beta^{2} r_{00}^{2}+64 b^{8} \beta r_{0} r_{00}+56 b^{6} \beta^{4} r_{00}^{2}+1888 n b^{6} \beta^{2} r_{00}^{2}+52 b^{8} \beta^{2} r_{00}^{2}-16 n b^{8} \beta r_{000}+92 b^{6} \beta^{2} r_{00}^{2}$ $+1404 n b^{2} \beta^{2} r_{0}^{2}+756 n b^{2} \beta^{2} r_{0} r_{00}-36 n b^{2} \beta^{2} r_{00}^{2}-14 n \beta^{4} r_{00}-288 \beta^{4} r_{00}^{2}-320 n \beta^{3} r_{0} r_{00}-68 n \beta^{3} r_{00}^{2}+240 b^{6} \beta r_{000}+256 r_{00}^{2} b^{6}$ $-72 b^{4} \beta^{2} r_{000}-76 n b^{4} \beta r_{000}-12 b^{4} \beta r_{0} r_{00}-24 b^{4} \beta r_{00}^{2}+56 n r_{00}^{2} b^{4}-96 b^{2} \beta^{3} r_{0}-16 b^{2} \beta^{3} r_{000}-46 n b^{2} \beta^{2} b^{i} r_{i 00}-12 n b^{2} \beta^{2} r_{0}$ $+1056 b^{2} \beta^{2} r_{0}^{2}+384 b^{2} \beta^{2} r_{0} r_{00}+124 b^{2} \beta^{2} r_{00}^{2}-460 n b^{2} \beta r_{0} r_{00}-84 n b^{2} \beta r_{00}^{2}+38 r_{00}^{2}-64 n \beta^{3} r_{0}+18 n \beta^{3} r_{000}-248 \beta^{3} r_{0} r_{00}$ $-148 n \beta^{2} r_{00}^{2}+110 b^{4} \beta r_{000}+636 r_{00}^{2} b^{4}-186 b^{2} \beta^{2} b^{i} r_{i 00}-140 b^{2} \beta^{2} r_{0}-16 b^{2} \beta^{2} r_{00}-36 b^{2} \beta^{2} r_{000}-14 n b^{2} \beta r_{000}-298 b^{2} \beta r_{0} r_{00}$ $+18 \beta^{3} r_{000}-75 n \beta^{2} b^{i} r_{i 00}-22 n \beta^{2} r_{0}-8 n \beta^{2} r_{00}-6 n \beta^{2} r_{000}+552 \beta^{2} r_{0}^{2}-8 \beta^{2} r_{00}-148 \beta^{2} r_{00}^{2}-86 n \beta r_{0} r_{00}-14 n \beta r_{00}^{2}$ $-60 \beta^{2} b^{i} r_{i 00}-14 \beta r_{00}^{2}-6 \beta^{2} r_{000}+28 \beta r_{0} r_{00}+38 n r_{00}^{2}-48 b^{6} \beta^{2} r_{000}-128 b^{4} \beta^{3} r_{000}+156 \beta^{2} r_{0} r_{00}+162 n \beta^{2} r_{0} r_{00}-22 \beta^{2} r_{0}$ $+232 b^{2} \beta^{3} r_{0} r_{00}-62 n b^{6} \beta^{4} r_{00}-96 n b^{8} \beta^{2} r_{000}+432 n b^{6} \beta^{2} r_{0} r_{00}+316 b^{2} r_{00}^{2}-48 n b^{2} \beta^{2} r_{000}+256 n b^{2} r_{00}^{2}-68 \beta^{3} r_{00}^{2}$ $-48 b^{2} \beta r_{00}^{2}+48 b^{4} \beta^{2} r_{00}+640 b^{8} \beta^{2} r_{00}-96 b^{6} \beta^{2} r_{0} r_{00}+108 n b^{4} \beta^{2} r_{0} r_{00}+12 b^{6} \beta^{2} r_{00}-14 n b^{4} \beta^{2} r_{000}+16 b^{2} \beta r_{000}$ $\left.-16 \beta^{3} r_{0}-312 b^{4} \beta^{2} r_{0}-64 n b^{2} \beta^{2} r_{00}+342 n \beta^{2} r_{0}^{2}\right)$, 
$A_{11}:=-\beta^{2}\left(336 n b^{8} \beta^{3} r_{000}-512 n b^{6} \beta^{4} r_{00}^{2}-176 n b^{8} \beta^{2} r_{00}^{2}-672 n b^{6} \beta^{4} r_{00}-560 b^{6} \beta^{4} r_{00}^{2}-1008 n b^{6} \beta^{3} r_{0} r_{00}+64 n b^{6} \beta^{3} r_{00}^{2}\right.$ $+336 b^{8} \beta^{3} r_{000}+80 b^{8} \beta^{2} b^{i} r_{i 00}+64 n b^{8} \beta^{2} r_{0}-128 n b^{8} \beta^{2} r_{00}+32 n b^{8} \beta^{2} r_{000}-128 n b^{8} \beta r_{0} r_{00}-672 b^{6} \beta^{4} r_{00}+128 n b^{6} \beta^{3} r_{0}$ $-12 b^{8} \beta^{2} r_{00}^{2}-108 b^{6} \beta^{3} r_{0} r_{00}+64 b^{6} \beta^{3} r_{00}^{2}-240 n b^{6} \beta^{2} r_{0}^{2}-24 \beta r_{000}-70 r_{00}^{2}-20 n b^{6} \beta^{2} r_{0} r_{00}-20 n b^{6} \beta^{2} r_{00}^{2}-24 n b^{4} \beta^{4} r_{00}$ $-2208 n b^{4} \beta^{3} r_{0} r_{00}+1152 n b^{4} \beta^{3} r_{00}^{2}+528 n b^{2} \beta^{4} r_{00}^{2}+64 b^{10} \beta r_{000}+128 n b^{8} r_{00}^{2}+80 b^{8} \beta^{2} b^{i} r_{i 00}+64 b^{8} \beta^{2} r_{0}-128 b^{8} \beta^{2} r_{00}$ $+176 n b^{8} \beta r_{000}-128 b^{8} \beta r_{0} r_{00}+128 b^{6} \beta^{3} r_{00}+888 b^{6} \beta^{3} r_{000}+704 n b^{6} \beta^{2} r_{0}-160 n b^{6} \beta^{2} r_{00}-176 n b^{6} \beta^{2} r_{000}-240 b^{6} \beta^{2} r_{0}^{2}$ $+1392 n b^{6} \beta r_{0} r_{00}+32 n b^{6} \beta r_{00}^{2}-528 b^{4} \beta^{4} r_{00}+1536 n b^{4} \beta^{3} r_{0}+576 n b^{4} \beta^{3} r_{00}+42 n b^{4} \beta^{3} r_{000}-768 b^{4} \beta^{3} r_{0} r_{00}+384 b^{4} \beta^{3} r_{00}^{2}$ $+936 n b^{4} \beta^{2} r_{00}^{2}+576 n b^{2} \beta^{4} r_{00}-480 b^{2} \beta^{4} r_{00}^{2}+2400 n b^{2} \beta^{3} r_{0} r_{00}+1104 n b^{2} \beta^{3} r_{00}^{2}+128 n \beta^{4} r_{00}^{2}+512 b^{8} \beta r 000-544 b^{8} r_{00}^{2}$ $-928 b^{6} \beta^{2} r_{00}-320 b^{6} \beta^{2} r_{000}-24 n b^{6} \beta r_{000}-1536 b^{6} \beta r_{0} r_{00}+1056 n b^{4} \beta^{2} b^{i} r_{i 00}-64 b^{6} \beta r_{00}^{2}-272 n b^{6} r_{00}^{2}+384 b^{4} \beta^{3} r_{0}$ $+960 n b^{4} \beta^{2} r_{0}-768 n b^{4} \beta^{2} r_{00}-312 n b^{4} \beta^{2} r_{000}-456 b^{4} \beta^{2} r_{0}^{2}+936 b^{4} \beta^{2} r_{0} r_{00}-792 b^{4} \beta^{2} r_{00}^{2}+2472 n b^{4} \beta r_{0} r_{00}-144 n b^{4} \beta r_{00}^{2}$ $+62 n b^{2} \beta^{3} r_{00}-300 n b^{2} \beta^{3} r_{000}+1104 b^{2} \beta^{3} r_{0} r_{00}+696 b^{2} \beta^{3} r_{00}^{2}-1584 n b^{2} \beta^{2} r_{0}^{2}+24 n b^{2} \beta^{2} r_{0} r_{00}+62 n b^{2} \beta^{2} r_{00}^{2}+240 n \beta^{4} r_{00}$ $+64 b^{6} r_{00}^{2}+48 b^{6} \beta^{2} b^{i} r_{i 00}+46 b^{4} \beta^{2} r_{0}-888 b^{4} \beta^{2} r_{00}-384 b^{4} \beta^{2} r_{000}-220 n b^{4} \beta r_{000}+912 b^{4} \beta r_{0} r_{00}-20 b^{4} \beta r_{00}^{2}-64 n b^{4} r_{00}^{2}$ $+272 n \beta^{3} r_{00}^{2}+408 b^{6} \beta r_{000}+1248 n \beta^{3} r_{0} r_{00}+1440 n b^{2} \beta^{3} r_{0}+360 b^{4} \beta^{3} r_{00}+160 n \beta^{3} r_{00}+70 \beta^{2} r_{0}-24 n b^{4} \beta^{2} r_{0} r_{00}$ $+128 b^{6} \beta^{3} r_{0}+1416 n b^{6} \beta^{3} r_{000}+64 n b^{10} \beta r_{000}-192 b^{2} \beta^{3} r_{000}+400 n b^{2} \beta^{2} b^{i} r_{i 00}+464 n b^{2} \beta^{2} r_{0}-102 n \beta^{3} r_{000}-44 n \beta r_{00}^{2}$ $-784 n b^{2} \beta^{2} r_{00}-164 n b^{2} \beta^{2} r_{000}-1392 b^{2} \beta^{2} r_{0}^{2}+624 b^{2} \beta^{2} r_{0} r_{00}-436 b^{2} \beta^{2} r_{00}^{2}+1436 n b^{2} \beta r_{0} r_{00}-168 n b^{2} \beta r_{00}^{2}+216 \beta^{4} r_{00}$ $+1320 \beta^{3} r_{0} r_{00}+26 \beta^{3} r_{00}^{2}-30 n \beta^{2} r_{0}^{2}+10 n \beta^{2} r_{0} r_{00}-28 n \beta^{2} r_{00}^{2}-40 b^{4} \beta r_{000}+684 b^{4} r_{00}^{2}+112 b^{2} \beta^{2} b^{i} r_{i 00}+320 b^{2} \beta^{2} r_{0}$ $+2288 b^{2} \beta r_{0} r_{00}-192 b^{2} \beta r_{00}^{2}-380 n b^{2} r_{00}^{2}+496 \beta^{3} r_{0}+160 \beta^{3} r_{00}-96 \beta^{3} r_{000}+47 n \beta^{2} b^{i} r_{i 00}+76 n \beta^{2} r_{0}-212 n \beta^{2} r_{00}$ $-23 \beta^{2} r_{00}^{2}+28 n \beta r_{0} r_{00}-110 b^{2} \beta r_{000}-32 b^{2} r_{00}^{2}+35 \beta^{2} b^{i} r_{i 00}-212 n \beta^{2} r_{00}-28 \beta^{2} r_{000}-24 n \beta r_{000}+72 \beta r_{0} r_{00}-44 n \beta r_{00}^{2}$ $+352 n \beta^{3} r_{0}-176 b^{2} \beta^{2} r_{000}-760 b^{2} \beta^{2} r_{00}+224 b^{6} \beta^{2} r_{0}-70 n r_{00}^{2}-134 n b^{2} \beta r_{000}-28 n \beta^{2} r_{000}+928 n b^{6} \beta^{2} b^{i} r_{i 00}-612 \beta^{2} r_{0}^{2}$ $+100 \beta^{2} r_{0} r_{00}-464 b^{6} \beta^{2} r_{00}^{2}+528 b^{2} \beta^{3} r_{00}+12 b^{2} \beta^{3} r_{0}-96 b^{2} \beta^{4} r_{00}+104 \beta^{4} r_{00}^{2}+480 b^{4} \beta^{3} r_{00}-32 b^{6} \beta^{2} b^{i} r_{i 00}$ $\left.-64 b^{8} \beta^{2} r_{000}-144 b^{4} \beta^{4} r_{00}^{2}-64 n b^{6} \beta^{3} r_{00}-3168 n b^{4} \beta^{4} r_{00}^{2}+176 b^{6} \beta^{2} r_{0} r_{00}-208 n b^{4} \beta^{2} r_{0}^{2}\right)$,

$A_{10}:=\beta^{3}\left(-192 n b^{6} \beta^{4} r_{00}^{2}+272 n b^{8} \beta^{3} r_{000}-144 n b^{8} \beta^{2} r_{00}^{2}-448 n b^{6} \beta^{4} r_{00}-560 n b^{6} \beta^{3} r_{0} r_{00}+32 n b^{6} \beta^{3} r_{00}^{2}-408 n b^{4} \beta^{4} r_{00}^{2}\right.$ $+16 n b^{8} \beta^{2} b^{i} r_{i 00}+32 n b^{8} \beta^{2} r_{0}-12 n b^{8} \beta^{2} r_{00}-64 n b^{8} \beta^{2} r_{000}+96 b^{8} \beta^{2} r_{00}^{2}-128 n b^{8} \beta r_{0} r_{00}-54 b^{6} \beta^{4} r_{00}+128 n b^{6} \beta^{3} r_{0}$ $-104 b^{6} \beta^{3} r_{0} r_{00}+32 b^{6} \beta^{3} r_{00}^{2}-48 n b^{6} \beta^{2} r_{0}^{2}+20 n b^{6} \beta^{2} r_{0} r_{00}-768 n b^{6} \beta^{2} r_{00}^{2}-52 n b^{4} \beta^{4} r_{00}+228 b^{4} \beta^{4} r_{00}^{2}+92 n b^{4} \beta^{3} r_{0} r_{00}$ $+16 b^{10} \beta r_{000}+12 b^{8} \beta^{2} b^{i} r_{i 00}+32 b^{8} \beta^{2} r_{0}-32 b^{8} \beta^{2} r_{00}-64 b^{8} \beta^{2} r_{000}-32 b^{8} \beta r_{0} r_{00}-16 n b^{8} r_{00}^{2}+18 b^{6} \beta^{3} r_{0}+128 b^{6} \beta^{3} r_{00}$ $-26 n b^{6} \beta^{2} b^{i} r_{i 00}+26 n b^{6} \beta^{2} r_{0}-64 n b^{6} \beta^{2} r_{00}-88 n b^{6} \beta^{2} r_{000}+144 b^{6} \beta^{2} r_{0} r_{00}+3168 b^{6} \beta^{2} r_{00}^{2}+64 n b^{6} \beta r_{0} r_{00}-96 n b^{6} \beta r_{00}^{2}$ $+1440 n b^{4} \beta^{3} r_{00}-336 b^{6} \beta^{2} r_{0}^{2}-60 n b^{4} \beta^{3} r_{000}-28 b^{4} \beta^{3} r_{0} r_{00}-432 b^{4} \beta^{3} r_{00}^{2}+56 n b^{4} \beta^{2} r_{0}^{2}+316 n b^{4} \beta^{2} r_{0} r_{00}+256 n b^{4} \beta^{2} r_{00}^{2}$ $+3924 n b^{2} \beta^{3} r_{0} r_{00}+96 n b^{2} \beta^{3} r_{00}^{2}-1332 n \beta^{4} r_{00}^{2}+88 b^{8} \beta r_{000}-256 b^{8} r_{00}^{2}+680 b^{6} \beta^{2} b^{i} r_{i 00}-224 b^{6} \beta^{2} r_{0}+128 b^{6} \beta^{2} r_{00}$ $-1928 b^{6} \beta r_{0} r_{00}+366 n b^{6} r_{00}^{2}+12 b^{4} \beta^{3} r_{00}+48 b^{4} \beta^{3} r_{000}-1704 n b^{4} \beta^{2} b^{i} r_{i 00}-96 n b^{4} \beta^{2} r_{0}-282 n b^{4} \beta^{2} r_{00}-116 n b^{4} \beta^{2} r_{000}$ $+330 b^{4} \beta^{2} r_{00}^{2}-154 n b^{4} \beta r_{0} r_{00}-816 n b^{4} \beta r_{00}^{2}+52 b^{2} \beta^{4} r_{00}-288 n b^{2} \beta^{3} r_{0}+912 n b^{2} \beta^{3} r_{00}-20 n b^{2} \beta^{3} r_{000}-1080 b^{2} \beta^{3} r_{0} r_{00}$ $+3636 n b^{2} \beta^{2} r_{0} r_{00}-1836 n b^{2} \beta^{2} r_{00}^{2}-632 n \beta^{4} r_{00}-1200 \beta^{4} r_{00}^{2}+44 n \beta^{3} r_{0} r_{00}-176 n \beta^{3} r_{00}^{2}+976 b^{6} \beta r_{000}+1360 r_{00}^{2} b^{6}$ $-528 b^{4} \beta^{2} r_{000}-100 n b^{4} \beta r_{000}-3504 b^{2} \beta r_{0} r_{00}-24 b^{4} \beta r_{00}^{2}+6840 n r_{00}^{2} b^{4}-96 b^{2} \beta^{3} r_{0}+240 b^{2} \beta^{6} r_{00}+16 b^{2} \beta^{3} r_{000}$ $-2272 n b^{2} \beta^{2} r_{00}-572 n b^{2} \beta^{2} r_{000}+600 b^{2} \beta^{2} r_{0}^{2}+1992 b^{2} \beta^{2} r_{0} r_{00}-588 b^{2} \beta^{2} r_{00}^{2}-1480 n b^{2} \beta r_{0} r_{00}-744 n b^{2} \beta r_{00}^{2}-608 \beta^{4} r_{00}$ $+1176 n b^{2} \beta^{4} r_{00}^{2}+1312 n b^{6} \beta^{3} r_{000}+320 b^{8} \beta^{3} r_{000}-60 b^{4} \beta^{2} r_{0}+624 b^{2} \beta^{4} r_{00}^{2}+960 n b^{4} \beta^{3} r_{0}-336 b^{6} \beta^{2} r_{0}^{2}+160 n b^{6} \beta r_{000}$ $+2556 n b^{2} \beta^{2} r_{0}^{2}+40 b^{4} \beta^{2} r_{0} r_{00}+32 n \beta^{3} r_{000}+112 n \beta^{3} r_{00}+128 \beta^{3} r_{0} r_{00}-188 \beta^{3} r_{00}^{2}+128 n \beta^{2} r_{0}^{2}+106 n \beta^{2} r_{0} r_{00}-164 n \beta^{2} r_{00}^{2}$ $+1028 b^{4} \beta r_{000}+4632 r_{00}^{2} b^{4}-196 b^{2} \beta^{2} b^{i} r_{i 00}-416 b^{2} \beta^{2} r_{0}-1120 b^{2} \beta^{2} r_{00}+14 \beta r_{000}-392 b^{2} \beta^{2} r_{000}-64 n b^{2} \beta r_{000}$ $+88 \beta^{3} r_{00}+44 \beta^{3} r_{000}-359 n \beta^{2} b^{i} r_{i 00}-82 n \beta^{2} r_{0}-500 n \beta^{2} r_{00}-94 n \beta^{2} r_{000}+1056 \beta^{2} r_{0} r_{00}-1392 \beta^{2} r_{00}^{2}-328 n \beta r_{0} r_{00}$ $-206 \beta^{2} b^{i} r_{i 00}-88 \beta^{2} r_{0}-46 \beta^{2} r_{00}-88 \beta^{2} r_{000}-7 n \beta r_{000}+680 \beta r_{0} r_{00}-132 n \beta r_{00}^{2}-192 b^{6} \beta^{4} r_{00}^{2}-276 b^{2} \beta r_{00}^{2}+404 n r_{00}^{2} b^{2}$ $-32 \beta^{3} r_{0}+352 n b^{8} \beta r_{000}+144 b^{4} \beta^{2} r_{00}+80 r_{00}^{2}+18 \beta^{2} r_{0}^{2}+308 b^{2} \beta r_{000}+356 r_{00}^{2} b^{2}-180 n \beta r_{00}^{2}-940 b^{2} \beta r_{0} r_{00}+92 n b^{4} \beta^{3} r_{00}^{2}$ $+128 n b^{6} \beta^{3} r_{00}+64 n b^{10} \beta r_{000}-1200 b^{4} \beta^{2} r_{0}^{2}-60 b^{2} \beta^{3} r_{00}^{2}+74 b^{4} \beta^{2} b^{i} r_{i 00}-1504 n b^{2} \beta^{2} b^{i} r_{i 00}-14 b^{6} \beta^{3} r_{000}+624 b^{4} \beta^{4} r_{00}$ $\left.+96 n b^{2} \beta^{4} r_{00}-224 b^{6} \beta^{2} r_{000}-368 n \beta^{3} r_{0}-272 n b^{2} \beta^{2} r_{0}+767 r_{00}^{2}\right)$, 
$A_{9}:=\beta^{4}\left(-2328 n b^{4} \beta^{4} r_{00}^{2}+32 n b^{8} \beta^{2} r_{000}-64 n b^{6} \beta^{3} r_{00}+936 n b^{6} \beta^{3} r_{000}-128 n b^{6} \beta^{2} r_{0} r_{00}-2592 n b^{6} \beta^{2} r_{00}^{2}-1536 n b^{4} \beta^{4} r_{00}\right.$ $-12 n b^{4} \beta^{3} r_{0} r_{00}+38 n b^{4} \beta^{3} r_{00}^{2}-144 n b^{2} \beta^{4} r_{00}^{2}+32 b^{8} \beta^{2} r_{000}+16 n b^{8} \beta r_{000}-160 n b^{8} r_{00}^{2}-64 b^{6} \beta^{3} r_{00}+102 b^{6} \beta^{3} r_{000}$ $-224 n b^{6} \beta^{2} r_{00}+34 n b^{6} \beta^{2} r_{000}-128 b^{6} \beta^{2} r_{0} r_{00}-2256 b^{6} \beta^{2} r_{00}^{2}+78 n b^{6} \beta r_{0} r_{00}+48 n b^{6} \beta r_{00}^{2}-182 b^{4} \beta^{4} r_{00}+960 n b^{4} \beta^{3} r_{0}$ $-256 b^{4} \beta^{3} r_{0} r_{00}+108 b^{4} \beta^{3} r_{00}^{2}-1080 n b^{4} \beta^{2} r_{0}^{2}-1416 n b^{4} \beta^{2} r_{0} r_{00}-4944 n b^{4} \beta^{2} r_{00}^{2}-240 n b^{2} \beta^{4} r_{00}-2160 b^{2} \beta^{4} r_{00}^{2}$ $+176 b^{8} \beta r_{000}+80 b^{8} r_{00}^{2}+576 b^{6} \beta^{2} b^{i} r_{i 00}+42 b^{6} \beta^{2} r_{0}-80 b^{6} \beta^{2} r_{00}-320 b^{6} \beta^{2} r_{000}-78 n b^{6} \beta r_{000}+40 b^{6} \beta r_{0} r_{00}-584 n b^{6} r_{00}^{2}$ $+152 b^{4} \beta^{3} r_{000}+2448 n b^{4} \beta^{2} r_{00}+1872 n b^{4} \beta^{2} r_{0}+1632 n b^{4} \beta^{2} r_{00}-240 n b^{4} \beta^{2} r_{000}-1560 b^{4} \beta^{2} r_{0}^{2}+504 b^{4} \beta^{2} r_{0} r_{00}$ $-720 b^{2} \beta^{4} r_{00}+4128 n b^{2} \beta^{3} r_{0}+1776 n b^{2} \beta^{3} r_{00}-756 n b^{2} \beta^{3} r_{000}+2280 b^{2} \beta^{3} r_{0} r_{00}+2304 b^{2} \beta^{3} r_{00}^{2}-3672 n b^{2} \beta^{2} r_{0}^{2}$ $+1440 \beta^{4} r_{00}^{2}+4440 n \beta^{3} r_{0} r_{00}+171 n \beta^{3} r_{00}^{2}+736 b^{6} \beta r_{000}+952 b^{6} r_{00}^{2}+1080 \beta^{4} r_{00}+864 b^{4} \beta^{2} b^{i} r_{i 00}+1080 b^{4} \beta^{2} r_{0}$ $+450 b^{4} \beta r_{0} r_{00}-240 b^{4} \beta r_{00}^{2}-104 n b^{4} r_{00}^{2}+2400 b^{2} \beta^{3} r_{0}+1296 b^{2} \beta^{3} r_{00}-432 b^{2} \beta^{3} r_{000}+182 n b^{2} \beta^{2} b^{i} r_{i 00}+154 n b^{2} \beta^{2} r_{0}$ $-3144 b^{2} \beta^{2} r_{0}^{2}+744 b^{2} \beta^{2} r_{0} r_{00}-2508 b^{2} \beta^{2} r_{00}^{2}+7212 n b^{2} \beta r_{0} r_{00}+12 n b^{2} \beta r_{00}^{2}+1824 n \beta^{3} r_{0}+952 n \beta^{3} r_{00}-504 n \beta^{3} r_{000}$ $+1644 n \beta^{2} r_{0} r_{00}+744 n \beta^{2} r_{00}^{2}-384 b^{4} \beta r_{000}+1908 b^{4} r_{00}^{2}+864 b^{2} \beta^{2} b^{i} r_{i 00}+1080 b^{2} \beta^{2} r_{0}-960 b^{2} \beta^{2} r_{00}-632 b^{2} \beta^{2} r_{000}$ $-312 b^{2} \beta r_{00}^{2}-6236 n t b^{2} t r_{00}^{2}+256 \beta^{3} r_{0}+904 \beta^{3} r_{00}-456 \beta^{3} r_{000}+384 n \beta^{2} b^{i} r_{i 00}+372 n \beta^{2} r_{0}-472 n \beta^{2} r_{00}$ $+166 n \beta r_{0} r_{00}+50 \beta^{2} r_{00}^{2}-54 n \beta r_{00}^{2}-740 b^{2} \beta r_{000}-44 b^{2} r_{00}^{2}+288 \beta^{2} r_{00}+3244 \beta^{2} r_{0}+13344 b^{2} \beta r_{0} r_{00}$ $+68 \beta^{2} r_{0} r_{00}+158 \beta^{3} r_{00}^{2}-104 n \beta^{2} r_{0}^{2}+1848 n \beta^{4} r_{00}^{2}+672 b^{4} \beta^{3} r_{00}+264 n b^{4} \beta r_{00}^{2}+1344 n \beta^{4} r_{00}+1296 n b^{4} \beta^{3} r_{000}$ $-888 b^{4} \beta^{2} r_{000}+2256 n b^{2} \beta^{2} r_{00}^{2}+8400 n b^{4} \beta r_{0} r_{00}+3624 n b^{2} \beta^{3} r_{00}^{2}-280 \beta^{2} r_{00}-136 \beta^{2} r_{000}-205 n \beta r_{000}+5376 \beta r_{0} r_{00}$ $-12 n r_{00}^{2}-193 \beta r_{000}-33 r_{00}^{2} b^{2} \beta^{2} b^{i} r_{i 00}+100 b^{2} \beta^{2} r_{0}-960 b^{2} \beta^{2} r_{00}-632 b^{2} \beta^{2} r_{000}-472 n \beta^{2} r_{00}+372 n \beta^{2} r_{0}-130 n \beta^{2} r_{000}$ $-1046 n b^{2} \beta r_{000}+13344 b^{2} \beta r_{0} r_{00}-312 b^{2} \beta r_{00}^{2}-62 n t b^{2} t r_{00}^{2}+226 \beta^{3} r_{0}+94 \beta^{3} r_{00}-46 \beta^{3} r_{000}+384 n \beta^{2} b^{i} r_{i 00}-136 \beta^{2} r_{000}$ $-2100 \beta^{2} r_{0}^{2}+68 \beta^{2} r_{0} r_{00}+504 \beta^{2} r_{00}^{2}+1656 n \beta r_{0} r_{00}-54 n \beta r_{00}^{2}-740 b^{2} \beta r_{000}-44 b^{2} r_{00}^{2}+288 \beta^{2} r_{00}+324 \beta^{2} r_{0}-280 \beta^{2} r_{00}$ $-96 \beta r_{00}^{2}-1225 n r_{00}^{2}-193 \beta r_{000}+5376 \beta r_{0} r_{00}-205 n \beta r_{000}-331 r_{00}^{2}+3432 n b^{2} \beta^{3} r_{0} r_{00}-28 n b^{4} \beta^{3} r_{00}+40 n b^{6} \beta^{2} b^{i} r_{i 00}$ $\left.-2736 b^{4} \beta^{4} r_{00}^{2}-840 b^{4} \beta^{2} r_{00}-456 n b^{2} \beta^{2} r_{0} r_{00}-42 n b^{2} \beta^{2} r_{000}-130 n \beta^{2} r_{000}-4704 b^{4} \beta^{2} r_{00}^{2}+90 b^{4} \beta^{3} r_{0}\right)$,

$A_{8}:=-\beta^{5}\left(-1056 n b^{4} \beta^{4} r_{00}^{2}+696 n b^{6} \beta^{3} r_{000}-1504 n b^{6} \beta^{2} r_{00}^{2}-720 n b^{4} \beta^{4} r_{00}-1344 b^{4} \beta^{4} r_{00}^{2}-336 n b^{4} \beta^{3} r_{0} r_{00}+672 n b^{4} \beta^{3} r_{00}^{2}\right.$ $-208 n b^{8} r_{00}^{2}+1032 b^{6} \beta^{3} r_{000}+96 n b^{6} \beta^{2} b^{i} r_{i 00}+192 n b^{6} \beta^{2} r_{0}-384 n b^{6} \beta^{2} r_{00}-480 n b^{6} \beta^{2} r_{000}-640 b^{6} \beta^{2} r_{00}^{2}+112 n b^{6} \beta r_{0} r_{00}$ $+576 n b^{4} \beta^{3} r_{0}+86 n b^{4} \beta^{3} r_{00}+144 n b^{4} \beta^{3} r_{000}-68 b^{4} \beta^{3} r_{0} r_{00}+720 b^{4} \beta^{3} r_{00}^{2}-216 n b^{4} \beta^{2} r_{0}^{2}+166 n b^{4} \beta^{2} r_{0} r_{00}-240 n b^{4} \beta^{2} r_{00}^{2}$ $+5676 n b^{2} \beta^{3} r_{0} r_{00}+4056 n b^{2} \beta^{3} r_{00}^{2}-1524 n \beta^{4} r_{00}^{2}-24 b^{8} \beta r_{000}+176 b^{8} r_{00}^{2}+648 b^{6} \beta^{2} b^{i} r_{i 00}+240 b^{6} \beta^{2} r_{0}+192 b^{6} \beta^{2} r_{00}$ $+184 b^{6} \beta r_{0} r 0_{0}-32 b^{6} \beta r_{00}^{2}-632 n b^{6} r_{00}^{2}+960 b^{4} \beta^{3} r_{0}+96 b^{4} \beta^{3} r_{00}+576 b^{4} \beta^{3} r_{000}-504 n b^{4} \beta^{2} b^{i} r_{i 00}+720 n b^{4} \beta^{2} r_{0}$ $+1536 b^{4} \beta^{2} r_{0} r_{00}+2472 b^{4} \beta^{2} r_{00}^{2}+2808 n b^{2} \beta r_{0} r_{00}-552 n b^{4} \beta r_{00}^{2}+336 b^{2} \beta^{4} r_{00}+1152 n b^{2} \beta^{3} r_{0}+3456 n b^{2} \beta^{3} r_{00}$ $+756 n b^{2} \beta^{2} r_{0}^{2}+7164 n b^{2} \beta^{2} r_{0} r_{00}-4680 n b^{2} \beta^{2} r_{00}^{2}-720 n \beta^{4} r_{00}-1632 \beta^{4} r_{00}^{2}+3084 n \beta^{3} r_{0} r_{00}+1212 n \beta^{3} r_{00}^{2}+528 b^{6} \beta r_{000}$ $-72 b^{4} \beta^{2} r_{0}-720 b^{4} \beta^{2} r_{00}-1368 b^{4} \beta^{2} r_{000}-288 n b^{4} \beta r_{000}-156 b^{4} \beta r_{0} r_{00}+52 r_{00}^{2}-264 b^{4} \beta r_{00}^{2}+154 n b^{4} r_{00}^{2}+1248 b^{2} \beta^{3} r_{0}$ $-1872 n b^{2} \beta^{2} b^{i} r_{i 00}+144 n b^{2} \beta^{2} r_{0}-630 n b^{2} \beta^{2} r_{00}-2304 n b^{2} \beta^{2} r_{000}-1536 b^{2} \beta^{2} r_{0}^{2}+4128 b^{2} \beta^{2} r_{0} r_{00}-3708 b^{2} \beta^{2} r_{00}^{2}$ $+1080 n \beta^{3} r_{00}-156 n \beta^{3} r_{000}+226 \beta^{3} r_{0} r_{00}+900 \beta^{3} r_{00}^{2}+1404 n \beta^{2} r_{0}^{2}+10 \beta r_{000}+3492 n \beta^{2} r_{0} r_{00}-5396 n \beta^{2} r_{00}^{2}+180 b^{4} \beta r_{000}$ $-360 b^{2} \beta^{2} r_{0}-264 b^{2} \beta^{2} r_{00}-1512 b^{2} \beta^{2} r_{000}-378 n b^{2} \beta r_{000}+308 b^{2} \beta r_{0} r_{00}-636 b^{2} \beta r_{00}^{2}+1750 n b^{2} r_{00}^{2}+384 \beta^{3} r_{0}+816 \beta^{3} r_{00}$ $-84 n \beta^{2} r_{0}-2736 n \beta^{2} r_{00}-546 n \beta^{2} r_{000}+984 \beta^{2} r_{0}^{2}+3408 \beta^{2} r_{0} r_{00}-4496 \beta^{2} r_{00}^{2}-244 n \beta r_{0} r_{00}-556 n \beta r_{00}^{2}+1140 b^{2} \beta r_{000}$ $-1992 \beta^{2} r_{00}-480 \beta^{2} r_{000}-87 n \beta r_{000}+4016 \beta r_{0} r_{00}-148 \beta r_{00}^{2}+4889 n r_{00}^{2}-432 n \beta^{3} r_{0}-816 \beta^{4} r_{00}+1728 b^{4} \beta^{2} b^{i} r_{i 00}$ $-798 n \beta^{2} b^{i} r_{i 00}+756 b^{2} \beta^{2} b^{i} r_{i 00}+384 n b^{6} \beta r_{000}-132 \beta^{2} r_{0}-264 b^{2} \beta^{4} r_{00}^{2}-1248 b^{4} \beta^{4} r_{00}+288 n b^{8} \beta r_{000}+72 b^{2} \beta^{3} r_{000}$ $-216 \beta^{2} b^{i} r_{i 00}-1392 b^{4} \beta^{2} r_{0}^{2}-1392 n b^{2} \beta r_{00}^{2}-612 n b^{2} \beta^{3} r_{000}-528 b^{6} \beta^{2} r_{000}+56 n b^{2} \beta^{4} r_{00}+16 n b^{6} \beta r_{00}^{2}-336 n b^{2} \beta^{4} r_{00}^{2}$ $+240 n b^{2} \beta r_{0} r_{00}+1248 b^{2} \beta^{3} r_{00}+404 b^{6} r_{00}^{2}-1416 b^{2} \beta^{3} r_{0} r_{00}-1536 n b^{4} \beta^{2} r_{00}-2664 n b^{4} \beta^{2} r_{000}+432 b^{2} \beta^{3} r_{00}^{2}+14704 b^{2} r_{00}^{2}$ $\left.-60 \beta^{3} r_{000}+1168 b^{4} r_{00}^{2}-96 \beta r_{00}^{2}+384 n b^{6} \beta^{2} r_{0}-160 n b^{4} \beta r_{000}-2100 \beta^{2} r_{0}^{2}-1046 n b^{2} \beta r_{000}+4512 \beta^{3} r_{0} r_{00}-72 n b^{2} \beta^{2} r_{00}\right)$, 
$A_{7}:=-\beta^{6}\left(-192 n b^{4} \beta^{3} r_{00}^{2}-1152 n b^{2} \beta^{4} r_{00}^{2}+240 n b^{6} \beta^{2} r_{000}-16 n b^{6} \beta r_{00}^{2}-384 n b^{4} \beta^{3} r_{00}+756 n b^{4} \beta^{3} r_{000}-192 b^{4} \beta^{3} r_{00}^{2}\right.$ $-288 n b^{2} \beta^{4} r_{00}-2448 b^{2} \beta^{4} r_{00}^{2}+1764 n b^{2} \beta^{3} r_{0} r_{00}+528 n b^{2} \beta^{3} r_{00}^{2}+4068 n \beta^{4} r_{00}^{2}+28 b^{6} \beta^{2} r_{000}-240 n b^{6} \beta r_{000}+32 b^{6} \beta r_{00}^{2}$ $+1080 n b^{4} \beta^{2} b^{i} r_{i 00}+864 n b^{4} \beta^{2} r_{0}+1056 n b^{4} \beta^{2} r_{00}+1008 n b^{4} \beta^{2} r_{000}+1044 b^{2} \beta^{3} r_{0} r_{00}-108 b^{2} \beta^{4} r_{00}+2904 b^{2} \beta^{3} r_{00}^{2}$ $-1152 b^{4} \beta^{2} r_{0} r_{00}-4320 b^{4} \beta^{2} r_{00}^{2}+4200 n b^{4} \beta r_{0} r_{00}+264 n b^{4} \beta r_{00}^{2}+2016 n b^{2} \beta^{3} r_{0}-240 n b^{2} \beta^{3} r_{00}-864 n b^{2} \beta^{3} r_{000}$ $-3132 n b^{2} \beta^{2} r_{0} r_{00}+2448 n \beta^{4} r_{00}+3420 \beta^{4} r_{00}^{2}+6120 n \beta^{3} r_{0} r_{00}+3120 n \beta^{3} r_{00}^{2}-408 b^{6} \beta r_{000}-3320 b^{6} r_{00}^{2}+1512 b^{4} \beta^{2} b^{i} r_{i 00}$ $+2232 b^{4} \beta^{2} r_{00}-288 b^{4} \beta^{2} r_{000}-3276 n b^{4} \beta r_{000}+6864 b^{4} \beta r_{0} r_{00}+192 b^{4} \beta r_{00}^{2}-30264 n b^{4} r_{00}^{2}+2880 b^{2} \beta^{3} r_{0}+1104 b^{2} \beta^{3} r_{00}$ $+2160 n b^{2} \beta^{2} r_{0}+5160 n b^{2} \beta^{2} r_{00}+72 n b^{2} \beta^{2} r_{000}-3060 b^{2} \beta^{2} r_{0}^{2}-1404 b^{2} \beta^{2} r_{0} r_{00}-1368 b^{2} \beta^{2} r_{00}^{2}+636 n b^{2} \beta r_{00}^{2}+2088 \beta^{4} r_{00}$ $+1704 n \beta^{3} r_{00}-1188 n \beta^{3} r_{000}+6624 \beta^{3} r_{0} r_{00}+2796 \beta^{3} r_{00}^{2}-2106 n \beta^{2} r_{0}^{2}-702 n \beta^{2} r_{0} r_{00}+6648 n \beta^{2} r_{00}^{2}-1224 b^{4} \beta r_{000}$ $+1728 b^{2} \beta^{2} r_{0}+2232 b^{2} \beta^{2} r_{00}-720 b^{2} \beta^{2} r_{000}-3258 n b^{2} \beta r_{000}+22416 b^{2} \beta r_{0} r_{00}+312 b^{2} \beta r_{00}^{2}-29736 n r_{00}^{2} b^{2}+3600 \beta^{3} r_{0}$ $+1053 n \beta^{2} b^{i} r_{i 00}+864 n \beta^{2} r_{0}+1344 n \beta^{2} r_{00}-186 n \beta^{2} r_{000}-302 \beta^{2} r_{0}^{2}-104 \beta r_{00}^{2}-1062 \beta^{2} r_{0} r_{00}+5040 \beta^{2} r_{00}^{2}+4740 n \beta r_{0} r_{00}$ $-7824 r_{00}^{2} b^{2}+837 \beta^{2} b^{i} r_{i 00}+756 \beta^{2} r_{0}+1368 \beta^{2} r_{00}-840 n \beta r_{000}+15216 \beta r_{0} r_{00}-7936 n r_{00}^{2}-2284 r_{00}^{2}-480 b^{4} \beta^{3} r_{00}$ $-2160 b^{2} \beta r_{000}-252 \beta^{2} r_{000}-1080 \beta^{3} r_{000}-744 \beta r_{000}+1944 b^{2} \beta^{2} b^{i} r_{i 00}-4332 n b^{4} \beta^{2} r_{00}^{2}+13092 n b^{2} \beta r_{0} r_{00}$ $-1620 n b^{2} \beta^{2} r_{0}^{2}-376 n r_{00}^{2} b^{6}-864 n b^{4} \beta^{2} r_{0} r_{00}+2808 n b^{2} \beta^{2} b^{i} r_{i 00}+97 b^{4} \beta^{3} r_{000}+3168 n \beta^{3} r_{0}-6876 r_{00}^{2} b^{4}$ $\left.+1536 \beta^{3} r_{00}+250 n \beta r_{00}^{2}+1080 b^{4} \beta^{2} r_{0}+5568 n b^{2} \beta^{2} r_{00}^{2}-540 b^{2} \beta^{3} r_{000}\right)$,

$A_{6}:=2 \beta^{7}\left(-288 n b^{2} \beta^{4} r_{00}^{2}+270 n b^{4} \beta^{3} r_{000}-1410 n b^{4} \beta^{2} r_{00}^{2}-864 b^{2} \beta^{4} r_{00}^{2}+180 n b^{6} \beta r_{000}+954 n b^{2} \beta^{3} r_{0} r_{00}+1260 n b^{2} \beta^{3} r_{00}^{2}\right.$ $-940 n r_{00}^{2} b^{6}+540 b^{4} \beta^{3} r_{000}+108 n b^{4} \beta^{2} b^{i} r_{i 00}+216 n b^{4} \beta^{2} r_{0}+888 n b^{4} \beta r_{0} r_{00}+48 n b^{4} \beta r_{00}^{2}-24 n b^{2} \beta^{2} r_{00}-68 n b^{2} \beta^{2} r_{000}$ $-162 n b^{2} \beta^{2} r_{0}^{2}+1674 n b^{2} \beta^{2} r_{0} r_{00}-144 b^{2} \beta^{4} r_{00}+432 n b^{2} \beta^{3} r_{0}+936 n b^{2} \beta^{3} r_{00}-324 n b^{2} \beta^{3} r_{000}+504 b^{2} \beta^{3} r_{0} r_{00}+154 b^{2} \beta^{3} r_{00}^{2}$ $+702 b^{4} \beta^{2} b^{i} r_{i 00}+32 b^{4} \beta^{2} r_{0}+684 b^{4} \beta^{2} r_{00}-816 n b^{2} \beta^{2} r_{00}^{2}+216 n \beta^{4} r_{00}-792 b^{4} \beta^{2} r_{000}+2718 n \beta^{3} r_{0} r_{00}+2250 n \beta^{3} r_{00}^{2}$ $-132 b^{4} \beta r_{00}^{2}-378 n b^{4} \beta r_{000}+2184 b^{4} \beta r_{0} r_{00}-1332 n b^{4} r_{00}^{2}+1008 b^{2} \beta^{3} r_{0}+1224 b^{2} \beta^{3} r_{00}-54 b^{2} \beta^{3} r_{000}-216 n b^{2} \beta^{2} b^{i} r_{i 00}$ $+2106 b^{2} \beta^{2} r_{0} r_{00}-235 b^{2} \beta^{2} r_{00}^{2}+2904 n b^{2} \beta r_{0} r_{00}-132 n b^{2} \beta r_{00}^{2}+36 \beta^{4} r_{00}+216 n \beta^{3} r_{0}+132 n \beta^{3} r_{00}-351 n \beta^{3} r_{000}$ $+2412 \beta^{3} r_{0} r_{00}+166 \beta^{3} r_{00}^{2}+12 n \beta^{2} r_{0}^{2}+2538 n \beta^{2} r_{0} r_{00}-2688 n \beta^{2} r_{00}^{2}+27 b^{4} \beta r_{000}+720 \beta^{3} r_{0}+504 b^{4} r_{00}^{2}+945 b^{4} \beta^{2} b^{i} r_{i 00}$ $-1044 b^{2} \beta^{2} r_{00}-1332 b^{2} \beta^{2} r_{000}-648 n b^{2} \beta r_{000}+5973 b^{2} \beta r_{0} r_{00}-366 b^{2} \beta r_{00}^{2}+11379 n b^{2} r_{00}^{2}+1044 \beta^{3} r_{00}-243 \beta^{3} r_{000}$ $+81 n \beta^{2} r_{0}-2148 n \beta^{2} r_{00}-729 n \beta^{2} r_{000}-288 \beta^{2} r_{0}^{2}+2538 \beta^{2} r_{0} r_{00}-2658 \beta^{2} r_{00}^{2}+74 n \beta r_{0} r_{00}-132 n \beta r_{00}^{2}+513 b^{2} \beta r_{000}$ $-1476 \beta^{2} r_{00}-630 \beta^{2} r_{000}-207 n \beta r_{000}+5640 \beta r_{0} r_{00}+120 \beta r_{00}^{2}+6337 n r_{00}^{2}+90 \beta r_{000}+7624 r_{00}^{2}-1782 n b^{2} \beta^{2} r_{000}$ $+135 \beta^{2} b^{i} r_{i 00}+27 \beta^{2} r_{0}-954 b^{2} \beta^{2} r_{0}^{2}+1736 b^{2} r_{00}^{2}-378 n \beta^{2} b^{i} r_{i 00}+216 b^{2} \beta^{2} r_{0}+432 n b^{2} \beta^{2} r_{0}-960 n b^{2} \beta^{2} r_{00}$ $\left.-306 b^{6} \beta r_{000}-48 b^{6} r_{00}^{2}-984 b^{4} \beta^{2} r_{00}^{2}+450 n \beta^{4} r_{00}^{2}\right)$,

$A_{5}:=3 \beta^{8}\left(68 n \beta^{4} r_{00}^{2}-288 n b^{2} \beta^{3} r_{00}^{2}+26 n b^{4} \beta^{2} r_{000}-32 n b^{4} \beta r_{00}^{2}+360 n \beta^{4} r_{00}-240 n b^{2} \beta^{3} r_{00}-18 n b^{2} \beta^{3} r_{000}-384 b^{2} \beta^{3} r_{00}^{2}\right.$ $+708 b^{2} \beta^{2} r_{00}^{2}+80 b^{4} \beta r_{00}^{2}-4684 n b^{4} r_{00}^{2}-384 b^{2} \beta^{3} r_{00}+1008 \beta^{4} r_{00}^{2}+900 n \beta^{3} r_{0} r_{00}+312 b^{4} \beta^{2} r_{000}-432 n b^{4} \beta r_{000}$ $-10 b^{2} \beta^{3} r_{000}+30 n b^{2} \beta^{2} b^{i} r_{i 00}+28 n b^{2} \beta^{2} r_{0}+108 n b^{2} \beta^{2} r_{00}+468 n b^{2} \beta^{2} r_{000}-1056 b^{2} \beta^{2} r_{0} r_{00}-20 n \beta^{2} r_{0}^{2}+132 b^{2} \beta^{2} r_{00}^{2}$ $+576 \beta^{4} r_{00}+432 n \beta^{3} r_{0}+24 n \beta^{3} r_{00}-432 n \beta^{3} r_{000}+1188 \beta^{3} r_{0} r_{00}+456 \beta^{3} r_{00}^{2}-738 n \beta^{2} r_{0} r_{00}+397 n \beta^{2} r_{00}^{2}-648 b^{4} \beta r_{000}$ $+576 b^{2} \beta^{2} b^{i} r_{i 00}+396 b^{2} \beta^{2} r_{0}+1824 b^{2} \beta^{2} r_{00}+168 b^{2} \beta^{2} r_{000}-1386 n b^{2} \beta r_{000}+4824 b^{2} \beta r_{0} r_{00}+272 b^{2} \beta r_{00}^{2}-16642 n b^{2} r_{00}^{2}$ $+1404 n \beta^{2} r_{00}+72 n \beta^{2} r_{000}-630 \beta^{2} r_{0}^{2}-978 \beta^{2} r_{0} r_{00}+3636 \beta^{2} r_{00}^{2}+2028 n \beta r_{0} r_{00}+106 n \beta r_{00}^{2}-1080 b^{2} \beta r_{000}-10370 b^{2} r_{00}^{2}$ $+396 \beta^{2} b^{i} r_{i 00}+306 \beta^{2} r_{0}+1452 \beta^{2} r_{00}+6 \beta^{2} r_{000}-585 n \beta r_{000}+6084 \beta r_{0} r_{00}-7573 n r_{00}^{2}-513 \beta r_{000}-3989 r_{00}^{2}$ $-28 \beta r_{00}^{2}+864 \beta^{3} r_{0}-6980 b^{4} r_{00}^{2}+396 n \beta^{2} b^{i} r_{i 00}-432 \beta^{3} r_{000}+240 \beta^{3} r_{00}+306 n \beta^{2} r_{0}-56 n b^{2} \beta^{2} r_{0} r_{00}$ $\left.+52 n b^{2} \beta r_{00}^{2}+1896 n b^{2} \beta r_{0} r_{00}\right)$,

$A_{4}:=9 \beta^{9}\left(+24 n b^{2} \beta^{3} r_{000}-9 n 6 \beta^{4} r_{00}^{2}+4 n b^{2} \beta^{2} r_{00}^{2}-48 n \beta^{4} r_{00}-192 \beta^{4} r_{00}^{2}-192 r_{00}^{2}-228 n \beta^{3} r_{0} r_{00}-264 n \beta^{3} r_{00}^{2}+12 n b^{4} \beta r_{000}\right.$ $+584 n b^{4} r_{00}^{2}+24 b^{2} \beta^{3} r_{000}-24 n b^{2} \beta^{2} b^{i} r_{i 00}-48 n b^{2} \beta^{2} r_{0}-80 n b^{2} \beta^{2} r_{00}+168 n b^{2} \beta^{2} r_{000}-144 b^{2} \beta^{2} r_{00}^{2}-316 n b^{2} \beta r_{0} r_{00}$ $-52 n b^{2} \beta r_{00}^{2}-96 \beta^{4} r_{00}-48 n \beta^{3} r_{0}-144 n \beta^{3} r_{00}+102 n \beta^{3} r_{000}-456 \beta^{3} r_{0} r_{00}-432 \beta^{3} r_{00}^{2}+18 n \beta^{2} r_{0}^{2}-234 n \beta^{2} r_{0} r_{00}$ $+1068 b^{4} r_{00}^{2}-150 b^{2} \beta^{2} b^{i} r_{i 00}-84 b^{2} \beta^{2} r_{0}-280 b^{2} \beta^{2} r_{00}+28 b^{2} \beta^{2} r_{000}-240 \beta^{3} r_{00}+192 n b^{2} \beta r_{000}-124 b^{2} \beta r_{0} r_{00}+36 b^{2} \beta r_{00}^{2}$ $+102 \beta^{3} r_{000}+15 n \beta^{2} b^{i} r_{i 00}-42 n \beta^{2} r_{0}+104 n \beta^{2} r_{00}+12 n \beta^{2} r_{000}+96 \beta^{2} r_{0}^{2}-372 \beta^{2} r_{0} r_{00}-36 \beta^{2} r_{00}^{2}-32 n \beta r_{0} r_{00}-124 n r_{00}^{2}$ $-74 n \beta r_{00}^{2}+148 b^{2} \beta r_{000}-868 b^{2} r_{00}^{2}-84 \beta^{2} r_{00}-1588 \beta r_{0} r_{00}+112 \beta^{2} r_{00}+186 \beta^{2} r_{000}+102 n \beta r_{000}-66 n \beta r_{00}^{2}$ $\left.-42 \beta^{2} r_{0}+28 \beta r_{000}+450 b^{2} r_{00}^{2}-184 n \beta^{2} r_{00}^{2}+202 b^{4} \beta r_{000}-144 \beta^{3} r_{0}\right)$, 


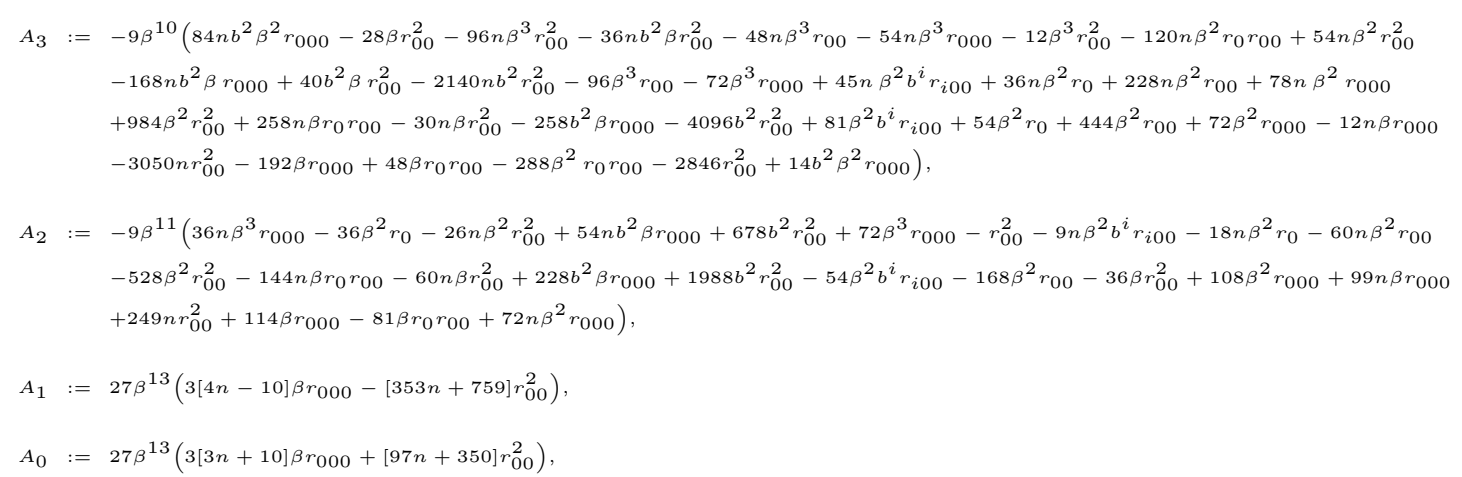

and

$$
\begin{aligned}
\tilde{X} & :=\frac{2}{\left(\alpha^{2}-\beta^{2}\right)\left(2 \alpha^{2} b^{2}+\alpha^{2}-3 \beta^{2}\right)^{3}}, \\
\tilde{Y} & :=\frac{2}{\alpha^{2}\left(\alpha^{2}-\beta^{2}\right)^{2}\left(2 b^{2} \alpha^{2}+\alpha^{2}-3 \beta^{2}\right)^{3}}, \\
\tilde{W} & :=-\frac{\alpha^{2}-4 \beta \alpha+\beta^{2}}{\left(2 b^{2} \alpha^{2}+\alpha^{2}-3 \beta^{2}\right)^{2}\left(\alpha^{2}-\beta^{2}\right)^{2}} .
\end{aligned}
$$

\section{References}

[1] Bácsó, S. and Matsumoto, M., On Finsler spaces of Douglas type, A generalization of notion of Berwald space. Publ. Math. Debrecen. 51(1997), 385-406.

[2] Bácsó, S. and Matsumoto, M., Finsler spaces with h-curvature tensor $H$ dependent on position alone. Publ. Math. Debrecen. 55(1999), 199-210.

[3] Berwald, L., Über die n-dimensionalen Geometrien konstanter Krümmung, in denen die Geraden die kürzesten sind. Math. Z. 30(1929), 449-469.

[4] Berwald, L., Über Parallelübertragung in Räumen mit allgemeiner Massbestimmung. Jber. Deutsch. Math.-Verein. 34(1926), $213-220$.

[5] Bidabad, B. and Tayebi, A., A classification of some Finsler connections. Publ. Math. Debrecen 71(2007), 253-260.

[6] Li, B. Shen, Y. and Shen, Z., On a class of Douglas metrics. Studia Sci. Math. Hungarica 46(3) (2009), 355-365.

[7] Matsumoto, M., An improvment proof of Numata and Shibata's theorem on Finsler spaces of scalar curvature. Publ. Math. Debrecen 64(2004), 489-500.

[8] Matsumoto, M., On the stretch curvature of a Finsler space and certain open problems. J. Nat. Acad. Math. India 11(1997), 22-32.

[9] Najafi, B. and Tayebi, A., Weakly stretch Finsler metrics. Publ Math Debrecen 7761(2017), 1-14.

[10] Szilasi, Z., On the projective theory of sprays with applications to Finsler geometry, PhD Thesis, Debrecen (2010), arXiv:0908.4384.

[11] Tayebi, A. Azizpour, E. and Esrafilian, E., On a family of connections in Finsler geometry. Publ. Math. Debrecen 72(2008), 1-15.

[12] Tayebi, A. and Najafi, B., Shen's processes on Finslerian connections. Bull. Iran. Math. Soc. 36(2) (2010), 57-73.

[13] Tayebi, A. and Najafi, B., Some curvature properties of $(\alpha, \beta)$-metrics. Bulletin Mathematique de la Societe des Sciences Mathematiques de Roumanie Tome 60 (108) No. 3, (2017), 277-291.

[14] Tayebi, A. and Najafi, B., On a class of homogeneous Finsler metrics. J. Geom. Phys. 140 (2019), 265-270.

[15] Tayebi, A. and Razgordani, M., Four families of projectively flat Finsler metrics with $\mathbf{K}=1$ and their non-Riemannian curvature properties. Rev. R. Acad. Cienc. Exactas Fís. Nat. Ser. A Math. RACSAM. 112(2018), 1463-1485.

[16] Tayebi, A. and Razgordani, M., On conformally flat fourth $\operatorname{root}(\alpha, \beta)$-metrics. Differ. Geom. Appl. 62(2019) 253-266.

[17] Tayebi, A. and Sadeghi, H., On Cartan torsion of Finsler metric. Publ. Math. Debrecen 82(2) (2013), 461-471.

[18] Tayebi, A. and Sadeghi, H., On a class of stretch metrics in Finsler geometry. Arabian Journal of Mathematics 8(2019), 153-160.

[19] Tayebi, A. and Tabatabeifar, T., Dougals-Randers manifolds with vanishing stretch tensor. Publ Math Debrecen 86(2015), 423-432.

[20] Tayebi, A. and Tabatabeifar, T., Unicorn metrics with almost vanishing $\mathbf{H}$ - and $\boldsymbol{\Xi}$-curvatures. Turkish J Math. 41(2017), 998-1008. 


\section{Affiliations}

AKBAR TAYEBI

ADDRESS: Department of Mathematics, Faculty of Science, University of Qom, Qom. Iran E-MAIL: akbar.tayebi@gmail.com

ORCID ID:0000-0002-6380-7624

NEDA IZADIAN

ADDRESS: Faculty of Mathematics and Computer Sciences

Shahid Chamran University of Ahvaz, Ahvaz. Iran

E-MAIL: nezadiyan@gmail.com

ORCID ID: 0000-0003-1110-2603 